Busse, A., and Sandham, N. (2012) Influence of an anisotropic slip-length boundary condition on turbulent channel flow. Physics of Fluids, 24 (5). 055111. ISSN 1070-6631

Copyright (C) 2012 American Institute of Physics

A copy can be downloaded for personal non-commercial research or study, without prior permission or charge

Content must not be changed in any way or reproduced in any format or medium without the formal permission of the copyright holder(s)

When referring to this work, full bibliographic details must be given

http://eprints.gla.ac.uk/84188

Deposited on: 08 August 2013

Enlighten - Research publications by members of the University of Glasgow http://eprints.gla.ac.uk 


\title{
Influence of an anisotropic slip-length boundary condition on turbulent channel flow
}

\author{
A. Busse ${ }^{1}$ and N. D. Sandham ${ }^{1}$ \\ Faculty of Engineering and the Environment, University of Southampton, Highfield, Southampton, \\ SO17 1BJ, United Kingdom
}

(Dated: 17 May 2012)

The effects of an anisotropic Navier slip-length boundary condition on turbulent channel flow are investigated parametrically by direct numerical simulations. The slip-length boundary condition is made direction dependent by specifying the value of the slip length independently for the streamwise and spanwise direction. The change in drag is mapped versus a wide range of streamwise and spanwise slip-length combinations at two different friction Reynolds numbers, $R e_{\tau_{0}}=180$ and $R e_{\tau_{0}}=360$. For moderate slip lengths both drag-reducing and drag-increasing slip-length combinations are found. The percentage drag increase saturates at approximately $60 \%$ for high spanwise slip. Once a threshold value for the streamwise slip length is exceeded, drag is reduced in all cases irrespective of the value of the spanwise slip length. The Reynolds number appears to have only little influence on the change in drag for the moderate Reynolds numbers studied here. A detailed comparison with the implicit theoretical formula of Fukagata et al. [Phys. Fluids 18, 051703 (2006)], which relates the change in drag with the streamwise and spanwise slip length, has been made. In general, this formula gives a fair representation of the change in drag; a modified version of this relation is presented, which improves the prediction for the change in drag for small slip length values and reduces the number of free parameters contained in the model. The effects of the slip-length boundary condition on the flow are further investigated using mean flow and turbulence statistics. For drag-neutral slip-length combinations the level of turbulent fluctuations is approximately unchanged. The presence of a slip-length boundary condition affects both the level of wall-shear stress fluctuations and the degree of intermittency of the wall-shear stress probability density function. The correlation statistics of the velocity field show that a high spanwise slip length causes a disruption of the near-wall streaks, while high streamwise slip favours an increasing streak regularity.

\section{INTRODUCTION}

Superhydrophobic surfaces have been the subject of intense research in recent years due to their potential to decrease skin-friction drag and their self-cleaning properties. A superhydrophobic surface combines a hydrophobic surface chemistry with structuring on micro- or nanoscales, resulting in very high contact angles typically in excess of 150 degrees $^{1}$. If a superhydrophobic surface is immersed in water an air-layer or air bubbles can be maintained on the surface ${ }^{1,2}$. Due to a lower shear stress at an air-water interface compared to a solid-water interface, superhydrophobic surfaces can influence the friction drag on a wall. The drag reducing properties of superhydrophobic surfaces have been demonstrated for both laminar and turbulent flow configurations (see for example Ref. 3).

The effect of a superhydrophobic surface on a flow is usually parametrised by a slip length in the form of a Navier slip-length boundary condition ${ }^{2} u_{\mathrm{s}}=\left.L \frac{\partial u}{\partial z}\right|_{\text {wall }}$, where $u_{s}$ is the slip velocity on the wall, $L$ the slip length and $z$ the wall normal direction. In recent years increasing slip length values up to the order of $100 \mu \mathrm{m}$ have been measured ${ }^{4}$. Although the application of superhydrophobic surfaces for drag reduction is still confined to microfluidic devices, this is a promising trend and superhydrophobic surfaces have the potential to reduce the drag at macroscopic scales in the future.

By structuring superhydrophobic surfaces the slip can be made direction dependent and give different values for the slip length in the streamwise $\left(L_{\mathrm{x}}\right)$ and spanwise directions of the flow $\left(L_{\mathrm{y}}\right)^{5}$. The effect of such a direction-dependent slip-length boundary condition on turbulent flow has been studied numerically by $\operatorname{Min} \& \mathrm{Kim}^{6}$, and later by Fukagata et $a l^{8}{ }^{8}$, for three different combinations: the purely streamwise slip case, where the slip-length boundary condition is applied to the streamwise component of the velocity only $\left(L_{\mathrm{x}} \neq 0, L_{\mathrm{y}}=0\right)$, the isotropic case with equal values for the stream- and spanwise $\operatorname{slip}$ length $\left(L_{\mathrm{x}}=L_{\mathrm{y}}\right)$ and the purely spanwise slip case $\left(L_{\mathrm{x}}=0, L_{\mathrm{y}} \neq 0\right)$. Drag reduction (compared to the reference case, a channel flow with standard no-slip boundary conditions) was found for the purely streamwise and isotropic slip cases whereas a drag increase was observed for the spanwise slip cases.

There remain several open questions on the effect of an anisotropic Navier-slip boundary condition on 
skin-friction drag. It would be of interest to know which $\left(L_{\mathrm{x}}, L_{\mathrm{y}}\right)$-combinations give no change in drag, that is the boundary between the drag-reducing and drag-increasing slip-length combinations. As the largest slip length value studied in Ref. 6 was 0.02 times the channel half-height $\left(L_{i}^{+_{0}}=L_{i} u_{\tau_{0}} / \nu \approx 3.6\right.$, the superscript $t_{0}$ indicates length scales given in units of the viscous length scale ${ }^{7}$ in the no-slip reference case in this paper), the effects of even higher slip lengths on the turbulence statistics are of interest as a limiting case. Also, the Reynolds number influence on the observed drag reduction is in need of further investigation; the previous investigation in Ref. 8 was limited to a small number of cases. Fukagata et al. ${ }^{8,9}$ derived an implicit formula for the relation between the change in drag and the streamwise and spanwise slip length in a turbulent channel flow. This relation was tested only for a small number of cases, and it is of interest to evaluate its general applicability. Finally, further turbulence statistics may give insight into how the turbulence is modified by the presence of a slip-length boundary condition. Here the statistics of the wallshear stress are of special interest, as it is directly involved in the specification of the slip-length boundary condition.

These questions will be addressed in this paper using direct numerical simulations (DNS) of turbulent channel flow to explore a wide range of $\left(L_{\mathrm{x}}, L_{\mathrm{y}}\right)$-combinations at two different Reynolds numbers, $R e_{\tau_{0}}=180$ and $R e_{\tau_{0}}=360$.

\section{NUMERICAL SETUP AND PARAMETER SPACE}

The incompressible Navier-Stokes equations, non-dimensionalised by the channel half-height $\delta$, the constant fluid density and the friction velocity in no-slip reference case $u_{\tau_{0}}$, were solved numerically for turbulent channel flow. A standard staggered-grid finite-difference code was employed using second-order central differences for the discretisation of the spatial derivatives and a second order Adams-Bashforth scheme for the time advancement. Periodic boundary conditions were applied in the streamwise and spanwise direction of the flow. The mass flow rate in the channel was kept constant by varying the mean streamwise pressure gradient in time, balancing the losses due to viscous dissipation (see e.g. Ref. 10). Simulations were conducted at two different Reynolds numbers $R e=\bar{U} \delta / \nu=2810$ and $R e=6250$ based on the mean streamwise velocity $\bar{U}$, the channel half-height $\delta$ and the kinematic viscosity $\nu$, or $R e_{\tau_{0}}=u_{\tau_{0}} \delta / \nu=180$ and $R e_{\tau_{0}}=360$ based on friction velocity $u_{\tau_{0}}$ in the reference case, which is a channel flow with standard no-slip boundary conditions on both walls.

For the lower Reynolds number a domain size of $10 \times 4 \times 2$ has been used where $x$ is the streamwise, $y$ the spanwise and $z$ the wall-normal direction of the channel. For the higher Reynolds number, $R e_{\tau_{0}}=360$, a reduced domain of size $5 \times 2 \times 2$ was used to limit the computational cost of the large number of simulations performed. The estimated change is drag is not affected significantly by the use of the smaller domain size (see appendix A).

For the $R e_{\tau_{0}}=180$ cases a grid of size $160 \times 128 \times 128$ has been employed, which was stretched in the $z$-direction in order to give a higher resolution near the wall $\left(\Delta z_{\min }^{+0}=0.75\right.$ and $\left.\Delta z_{\max }^{+0}=4.9\right)$. A grid of size $160 \times 128 \times 256$ was used for the $R e_{\tau_{0}}=360$ cases using the same grid-stretching in the $z$-direction and giving the same resolution in wall units.

As an initial state for the velocity field a fully developed turbulent velocity field from the corresponding reference simulation of turbulent channel flow with standard no-slip boundary conditions has been used. An initial Euler forward time integration step is used for the start-up of the Adams-Bashforth scheme.

The slip-length boundary condition

$$
u_{\mathrm{s}}=\left.L_{x} \frac{\partial u}{\partial z}\right|_{\text {wall }}, \quad v_{\mathrm{s}}=\left.L_{y} \frac{\partial v}{\partial z}\right|_{\text {wall }}
$$

is applied symmetrically to the upper and lower wall of the channel. Note that the Navier-slip boundary condition takes this simple form only for planar walls. For curved boundaries the Navier-slip boundary condition takes a more complicated form ${ }^{11}$.

In some cases combinations with a no-slip boundary condition, equivalent to $L_{i}=0$, or with a full-slip boundary condition, equivalent to an infinite slip length $L_{i}=\infty$, are studied. The investigated slip length values are given in table I and have been based on the viscous length scale $\nu / u_{\tau_{0}}$ of the respective no-slip reference cases. Simulations have been run for all 80 resulting combinations of the streamwise and spanwise slip lengths in the $R e_{\tau_{0}}=180$ case. Another 72 simulations were performed for the $R e_{\tau_{0}}=360$ case. In the sections V-VII the focus is on the results for the $R e_{\tau_{0}}=180$ cases. First, in section, the most important practical aspect, i. e. the change in drag, will be discussed. A comparison with the formula of Fukagata et 
TABLE I. The streamwise and spanwise slip length values used in the simulations. For both Reynolds numbers all possible combinations $\left(L_{x}^{+0}, L_{y}^{+o}\right)$ of the given values have been studied.

\begin{tabular}{lll}
\hline \hline$R e_{\tau_{0}}$ & dir & $L_{i}^{+0}$ \\
\hline 180 & $x$ & $0,0.1,0.316,1,3.16,10,31.6,100$ \\
& $y$ & $0,0.1,0.316,1,3.16,10,31.6,100,316, \infty$ \\
360 & $x$ & $0,0.1,0.316,1,3.16,10,31.6,100$ \\
& $y$ & $0,0.1,0.316,1,3.16,10,31.6,100, \infty$ \\
\hline \hline
\end{tabular}

(a)

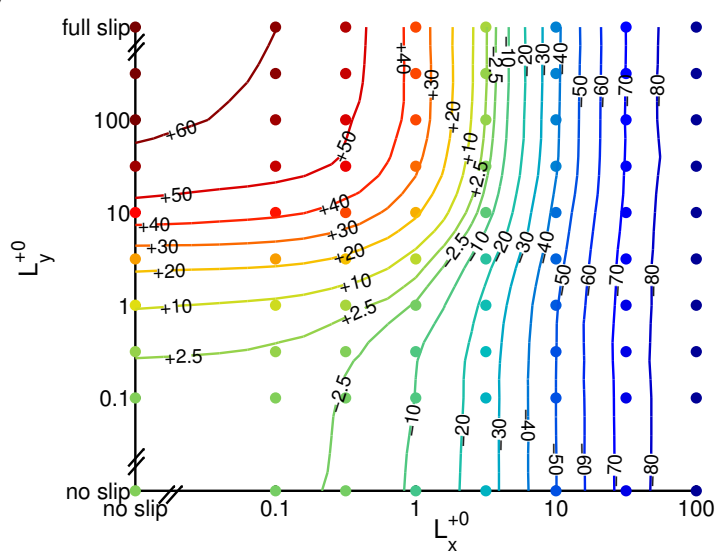

(b)

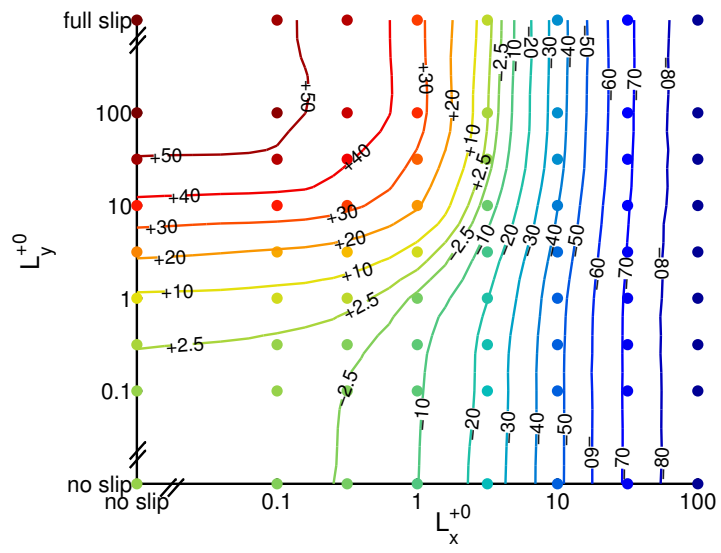

FIG. 1. The percentage change in drag versus the streamwise and spanwise slip length. (a) $R e_{\tau_{0}}=180 ;(\mathrm{b})$ $R e_{\tau_{0}}=360$. The large dots in the figures indicate the computed points on which the contour plot has been based. As the measurement of the change in drag is subject to measurement errors it is difficult to establish the exact location of the neutral curve in the contour plots of the change in drag. Therefore the $-2.5 \%$ and $+2.5 \%$ contours are shown instead.

$a .^{8}$, an implicit relation between change in drag and streamwise and spanwise slip length, is given in section IV where also an improved version of this formula will be presented. Section V focuses on the changes in the mean velocity profile and the profiles of the rms velocity fluctuations. The wall-shear stress statistics are discussed in section VI, and the changes in the flow structure are explained in section VII.

\section{CHANGE IN DRAG}

The effect of the slip-length boundary condition on the skin friction drag is measured by the percentage change in drag $\Delta \mathrm{D}$ defined by

$$
\Delta \mathrm{D}=\frac{\left\langle-\frac{d \bar{p}}{d x}\right\rangle-\left\langle-\left.\frac{d \bar{p}}{d x}\right|_{0}\right\rangle}{\left\langle-\left.\frac{d \bar{p}}{d x}\right|_{0}\right\rangle} \times 100
$$

Here, $\frac{d \bar{p}}{d x}$ is the (time-dependent) mean streamwise pressure gradient and $\left.\frac{d \bar{p}}{d x}\right|_{0}$ is the mean streamwise pressure gradient in the reference case. The percentage change in drag is greater than zero if the drag is increased and lower than zero for drag reduction. The values measured in the case of $R e_{\tau_{0}}=180$ are shown in figure 1 (a).

The same trends as reported by $\mathrm{Min} \mathscr{E} \mathrm{Kim}^{6}$ are recovered: a reduction in drag is observed in the purely streamwise and isotropic slip cases and a drag increase is observed for the purely spanwise slip case. Once the spanwise slip length is increased to values of the order of the channel half-height the increase in drag begins to saturate at approximately $60 \%$ in the purely spanwise slip case $\left(\Delta D \approx 63 \%\right.$ for $\left.L_{x}=0, L_{y}=\infty\right)$.

The transition from the drag-reducing to the drag-increasing domain is smooth. The neutral curve, i.e. the boundary between the drag-reducing and drag-increasing domain on the map, levels off as the streamwise 
slip length is increased. Once a streamwise slip length of approximately $L_{\mathrm{x}, \text { thresh }}^{+_{0}}=3.5$ is exceeded the drag is reduced in all cases. Even an infinite spanwise slip length cannot overcome the drag reducing effects. In the vast majority of cases therefore the drag will be reduced. As the streamwise slip length is further increased the turbulent fluctuations become weaker and weaker and the flow reverts slowly to the laminar case. This is the case for all simulations with $L_{x}^{+0}=100$ at $R e_{\tau_{0}}=180$. Since the process of relaminarisation is slow these cases have not fully laminarised by the time the simulations have been terminated. Linear stability analysis ${ }^{12}$ shows that streamwise slip delays transition to turbulence. It is therefore not unexpected that a reverse transition occurs for high streamwise slip lengths. An accurate boundary for the transition from the turbulent to the laminar case cannot be given here as this would require a different setup of the numerical simulations ${ }^{13,14}$, which would incur considerably higher computational expense.

The possible future application of superhydrophobic surfaces in macroscopic devices, e.g. for the coating of ship hulls, makes the Reynolds number dependence of the change in drag of particular interest, as these devices typically operate at Reynolds numbers much higher than those commonly encountered in microfluidic devices. In order to investigate the Reynolds number dependence of the results presented here a series of simulations using a reduced domain size has been run at $R e_{\tau_{0}}=360$. Except for the slip-length combinations with $L_{y}^{+0}=316$ the same range of slip-length combinations (based on the viscous length scale in the noslip reference case) has been covered. Going to even higher Reynolds numbers has not been attempted as this would entail a prohibitively high computational cost due to the large number of simulations needed to construct the slip length map. It was checked that the smaller domain size does not influence the estimated change in drag (see appendix A).

In figure 1 (b) the change in drag is shown for the $R e_{\tau_{0}}=360$ cases. The percentage change is the same or slightly lower than in the $R e_{\tau_{0}}=180$ cases (see also table IV). For moderate slip lengths the values of $\Delta D$ are close to the values obtained in the $R e_{\tau_{0}}=180$ case. For high spanwise slip lengths the change in drag is consistently lower than in the $R e_{\tau_{0}}=180$ case. For example, for the $\left(L_{x}^{+0}=0, L_{y}^{+0}=\infty\right)$ slip-length combination the change in drag $\Delta D \pm \sigma(\Delta D)$ is $55.5 \% \pm 4.8 \%$ in the $R e_{\tau_{0}}=360$ case compared to $63.2 \% \pm 3.7 \%$ in the $R e_{\tau_{0}}=180$ case. A similar reduction of the drag increase for high spanwise slip lengths with Reynolds number was also observed in Ref. 8.

The neutral curve is in approximately the same place as in the $R e_{\tau_{0}}=180$ case, implying that the critical streamwise slip length, $L_{\mathrm{x}, \text { thresh }}^{+0}$, is approximately the same in both cases, independent of the Reynolds number. In conclusion, we can not detect a significant Reynolds-number dependence of the results at moderate slip lengths, although a trend towards lower increases in drag for high spanwise slip lengths is observed.

\section{COMPARISON WITH A THEORETICAL PREDICTION FOR THE CHANGE IN DRAG}

Fukagata et al. ${ }^{8,9}$ have proposed a simple implicit formula for the relationship between the change in drag and the streamwise and spanwise slip length for turbulent channel flow

$$
\frac{1}{\kappa} \ln R e_{\tau_{0}}+F_{0}=\left(1-R_{D}\right) L_{x}^{+0}+\frac{\sqrt{1-R_{D}}}{\kappa} \ln \left(\sqrt{1-R_{D}} R e_{\tau_{0}}\right)+\sqrt{1-R_{D}} F\left(\sqrt{1-R_{D}} L_{y}^{+_{0}}\right) .
$$

Here, $R_{D}$ is the drag reduction rate $\left(\Delta D=-100 R_{D}\right), F_{0}=3.2$ is a constant taken from Ref. 15 and $F$ is an empirical function describing the changes in the mean streamwise velocity in the purely spanwise slip case $F\left(L_{y}^{+}\right)=\bar{U} u_{\tau}^{-1}-\kappa^{-1} \ln \left(R e_{\tau}\right)$, where $L_{y}^{+}=L_{y}^{+0} u_{\tau} / u_{\tau_{0}}=L_{y}^{+0} \sqrt{1-R_{D}}$ and $R e_{\tau}=\sqrt{1-R_{D}} R e_{\tau_{0}}$. Fukagata et al. ${ }^{8}$ proposed the following functional form for $F$

$$
F\left(L_{y}^{+}\right)=F_{\infty}+\left(F_{0}-F_{\infty}\right) \exp \left[-\left(L_{y}^{+} / a\right)^{b}\right],
$$

where $F_{\infty}=-0.8, a=7$ and $b=0.7$ were obtained from a fit to (their) DNS data. At the Reynolds numbers studied by Fukagata et al. ${ }^{8}, R e_{\tau_{0}}=180$ and $R e_{\tau_{0}}=400$, the fitting parameters showed no significant Reynolds number dependence. Fukagata et al. ${ }^{8}$ tested their model only for a small range of values for the purely streamwise, isotropic and purely spanwise slip cases. The comprehensive study of slip length combinations presented here enables a detailed evaluation of relation (3).

\section{A. Modification of the formula}

In the course of the comparison with relation (3) it became apparent that the choice of parameters $F_{0}$, $F_{\infty}$, and the form of the fitting function (4) made in Ref. 8 are not ideal and can be improved. Although 
(a)

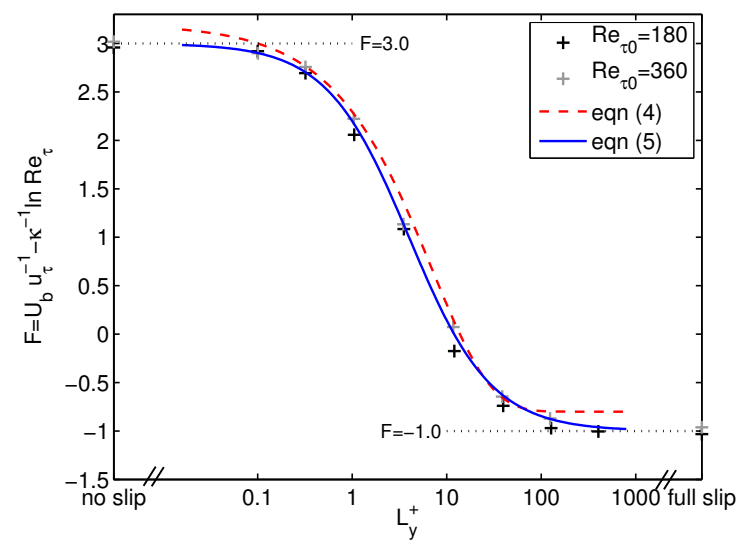

(b)

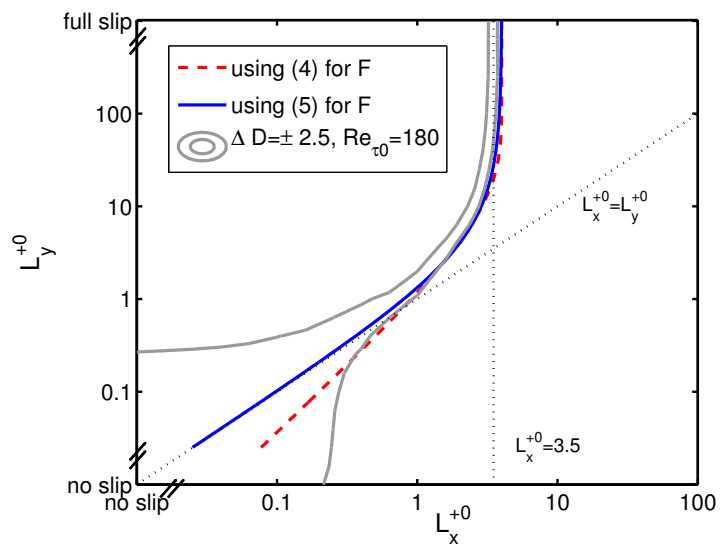

FIG. 2. (a): $F\left(L_{y}^{+}\right)$versus spanwise slip length $L_{y}^{+}$in the purely spanwise slip case. Two different versions for a function fitted to $F$ are shown. (b): The neutral curve for the original (4) and the new version (5) version of the fitting function $F$.

the modifications are small, they have a noticeable effect. In the following a motivation for the changes made will be given.

The first point is the constant $F_{0}$; in Ref. 8 this was set to the value 3.2 taken from Dean's formula for the 'optimum log-law for skin friction' ${ }^{15}$. No error bounds are given for $F_{0}$ in Ref. 15 , but it is calculated from two quantities, the Coles parameter II and the velocity defect ratio $J$, which both show significant experimental scatter. Computing $F_{0}$ from the present DNS data in the no-slip case and from independent DNS data for turbulent channel flow ${ }^{16}$ suggests that $F_{0}=3.0$ is a more suitable choice at the Reynolds numbers studied in this paper.

The second point relates to the choice of the fitting function, relation (4), i.e. its functional form and the involved fitting parameters $\left(F_{\infty}, a, b\right)$. The fitting function $F$ is used to capture the changes in the mean streamwise velocity in the purely spanwise slip case. The data points for $F=\bar{U} u_{\tau}^{-1}-\kappa^{-1} \ln \left(R e_{\tau}\right)$ are $\operatorname{shown}$ as a function of $L_{y}^{+}$in figure 2 (a). $F$ approximately follows the form of a smeared-out Heaviside function of $-\ln \left(L_{y}^{+}\right)$, i.e. for small slip length values $F$ is approximately constant, $F\left(L_{y}^{+}\right) \approx F_{0}$, for intermediate slip length values there is a rapid decrease in $F$ with increasing slip length, and for high spanwise slip lengths $F$ again levels at a constant value $F_{\infty}=F\left(L_{y}^{+} \rightarrow \infty\right)$. Fukagata et al. ${ }^{8}$ chose $F_{\infty}=-0.8$, whereas for our data $F_{\infty}$ can be computed directly from the $\left(L_{x}=0, L_{y}=\infty\right)$ combinations, giving $F_{\infty}=-1.03$ for the $R e_{\tau_{0}}=180$ case and $F_{\infty}=-0.96$ for $R e_{\tau_{0}}=360$. Since the difference between the two Reynolds numbers is small, $F_{\infty}=-1.0$ will be used in the following for both Reynolds numbers. Instead of the functional form for $F$ proposed by Fukagata et al. ${ }^{8}$ (4) we propose the following form for $F$

$$
F\left(L_{y}^{+}\right)=F_{\infty}+\frac{\left(F_{0}-F_{\infty}\right)^{2}}{\left(F_{0}-F_{\infty}\right)+L_{y}^{+}} .
$$

The new form of the fitting function has the advantage of being simpler than relation (4); the number of parameters is reduced from four $\left(F_{0}, F_{\infty}, a, b\right)$ to two $\left(F_{0}, F_{\infty}\right)$. The rms deviation of the data points for $F$ from the new fitting function (5) is reduced by more than a factor of 2 compared to the old version (4) (see also figure $2(\mathrm{a}))$. However, the crucial advantage of the new fitting function is visible at small values of the slip lengths as will be pertinent in the following discussion of the neutral curve.

\section{B. The location of the neutral curve}

From relation (3) a prediction can be made for the location of the neutral curve, i.e. the boundary between the drag-reducing and drag-increasing domain on the slip length map (see section III), by setting $R_{D}=0$ :

$$
L_{x}^{+o}\left(R_{D}=0, L_{y}^{+0}\right)=F_{0}-F\left(L_{y}^{+0}\right)
$$

where $F\left(L_{y}^{+0}\right)$ refers to the fitting function discussed above. The location and form of the neutral curve is therefore strongly influenced by the functional form of the fitting function $F$. The location of the neutral 
(a)

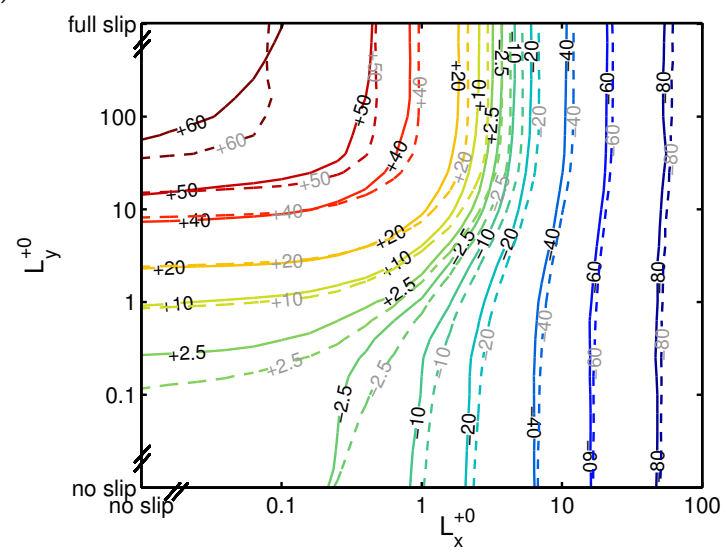

(b)

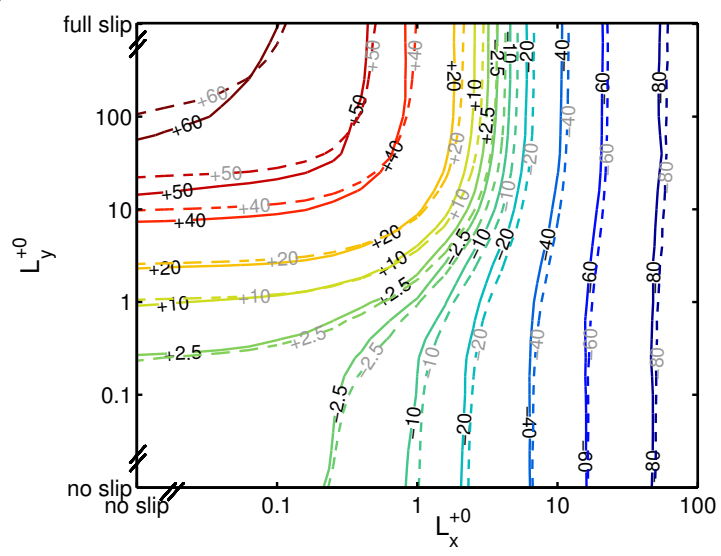

FIG. 3. The measured (continuous contours, black labels) and the predicted (dashed contours, grey labels) percentage change in drag. (a): using (4) for the fitting function $F$ in the theoretical prediction (3), (b): using relation (5) for the fitting function $F$. Case $R e_{\tau_{0}}=180$.

TABLE II. Differences between predicted $\Delta D_{\mathrm{P}}$ and measured (DNS) $\Delta D_{\mathrm{DNS}}$ values for the percentage change in drag $\Delta D$.

\begin{tabular}{llll}
\hline \hline$R e_{\tau_{0}}$ & form of $\mathrm{F}$ & $\left\langle\Delta D_{\mathrm{P}}-\Delta D_{\mathrm{DNS}}\right\rangle$ & $\left\langle\left(\Delta D_{\mathrm{P}}-\Delta D_{\mathrm{DNS}}\right)^{2}\right\rangle^{1 / 2}$ \\
\hline 180 & rel. (4) & 2.0 & 2.5 \\
180 & rel. (5) & 1.1 & 2.2 \\
360 & rel. (4) & 1.5 & 1.9 \\
360 & rel. (5) & 0.7 & 1.4 \\
\hline \hline
\end{tabular}

curve is shown in Figure 2 (b) for both the original (4) and the new (5) form of the fitting function $F$. For high values of the spanwise slip length $L_{y}^{+0}$ the two curves coincide. A clear difference is visible for small slip length values. Here, the neutral curve based on the new form of the fitting function $F$ gives a better representation. From our data we find that drag is reduced for all slip length combinations where $L_{x}^{+o} \geq L_{y}^{+0}$; similarly it was observed by $\mathrm{Min} \mathscr{E} \mathrm{Kim}^{6}$ in the isotropic case $L_{x}^{+o}=L_{y}^{+o}$ that the drag is reduced or, for very low values of the slip length, not changed at all. This implies that for the neutral curve $L_{x}^{+_{0}} \leq L_{y}^{+_{0}}$. As is apparent from figure $2(\mathrm{~b})$, the neutral curve based on the original form of the fitting function (4) predicts - contrary to these observations - that for small slip length values $L_{x}^{+o}$ is larger than $L_{y}^{+0}$ along the neutral curve. The new form of the fitting function however preserves the empirical inequality that for drag-neutral slip length combinations $L_{x}^{+0} \leq L_{y}^{+0}$.

In section III a critical threshold value was identified for the streamwise slip length, $L_{x, \text { thresh }}^{+0}=3.5$, beyond which drag is reduced in all cases. From relation (6) the following prediction for this threshold value can be obtained

$$
L_{x, \text { thresh }}^{+o}=L_{x}^{+_{0}}\left(R_{D}=0, L_{y}^{+_{0}} \rightarrow \infty\right)=F_{0}-F_{\infty}
$$

Note that the result is the same for both the new and the old form of the fitting function, giving $L_{x, \text { thresh }}^{+o}=4$, close to the value obtained from the present simulations.

As the relation for the neutral curve (6) contains no explicit Reynolds number dependence, the neutral curve and the threshold value $L_{x, \text { thresh }}^{+0}$ should not show any Reynolds number dependence (provided that the parameters used, i.e. $\left(F_{0}, F_{\infty}, a, b\right)$ or $\left(F_{0}, F_{\infty}\right)$, are Reynolds number independent). This agrees with the observation made in section III that the neutral curve is approximately same for both Reynolds numbers studied. 


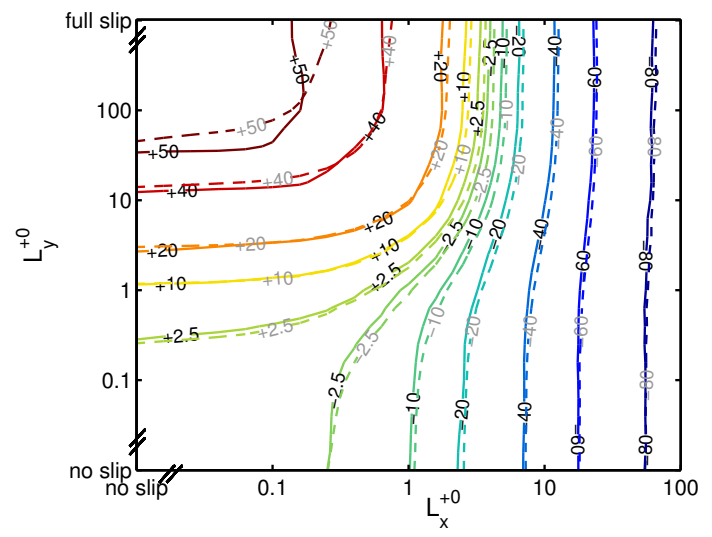

FIG. 4. The measured (continuous contours, black labels) and the predicted (dashed contours, grey labels) percentage change in drag $\Delta D$ using (5) for the fitting function $F$ in the theoretical prediction (3). Case $\operatorname{Re}_{\tau_{0}}=360$.

\section{Change in drag: prediction and measurements}

In figure 3 the change in drag obtained from the DNS data is compared with the predicted change in drag using the implicit relation (3). Results are shown for both using the original (4) and the new (5) version of the fitting function $F$. In table II errors for the predicted change in drag compared to the values for the DNS data are listed. Given its simple form, the prediction of Fukagata et al ${ }^{8}$ (3) makes a surprisingly good prediction for the change in drag. On average, the difference between the predicted percentage change in drag and the DNS results is approximately two percentage points (see table II). While there is little difference between the results for the original and the new version of the fitting function at high streamwise slip lengths, the accuracy of the prediction is significantly improved at small slip lengths by using the new form of the fitting function (5). As the fitting function is used to capture the effects of purely spanwise slip, this behaviour is expected.

For the new form of the fitting function the rms error for the change in drag is lower. Note that, for both old and new form of the fitting function and both $R e_{\tau_{0}}=180$ and $R e_{\tau_{0}}=360$, there is a bias for the theoretical prediction to overpredict $\Delta D$ (see table II). This means that the drag increase with spanwise slip length tends to be overpredicted whereas the drag reduction with increasing streamwise slip length tends to be underpredicted by the theoretical relation.

A good prediction in the change in drag is also achieved at the higher Reynolds number $R e_{\tau_{0}}=360$ (see figure 4). Here, the new form of the fitting function also improves the results compared to the original form (see table II). Relation (3) predicts a weakening of the drag increase caused by the spanwise slip length $L_{y}^{+0}$ with increasing Reynolds number. This is mirrored in our data (see section III).

In conclusion of this section, a surprisingly good prediction for the change in drag has been obtained from the relation of Fukagata et al. ${ }^{8}$, which has been further improved and simplified by a change of the fitting function $F$. In the derivation of their relation Fukagata et al. ${ }^{8}$ have simply added the effects of streamwise and spanwise slip, and have not considered any coupling between streamwise and spanwise slip, i.e. cross terms in $L_{x}^{+_{0}}$ and $L_{y}^{+_{0}}$. As their approach is successful overall, the potential coupling between streamwise and spanwise slip must be too weak to have a strong effect on the change in drag.

\section{VELOCITY PROFILES}

The changes in the mean streamwise velocity profile and the turbulent velocity fluctuations will be first discussed using the shape factor $H$, the centreline velocity, the mean streamwise slip velocity on the walls and the peak value of the rms streamwise velocity fluctuations in order to give an overview over all cases on the slip length map. In the second part of this section details in the profiles will be discussed for two different routes across the slip length map, which are of special interest regarding the transition from the drag-increasing to the drag-reducing domain of the slip length map.

The spanwise slip length has little influence on the mean streamwise slip velocity (see figure 5) and as expected the slip velocity on the wall increases with increasing streamwise slip length. The shape factor, 


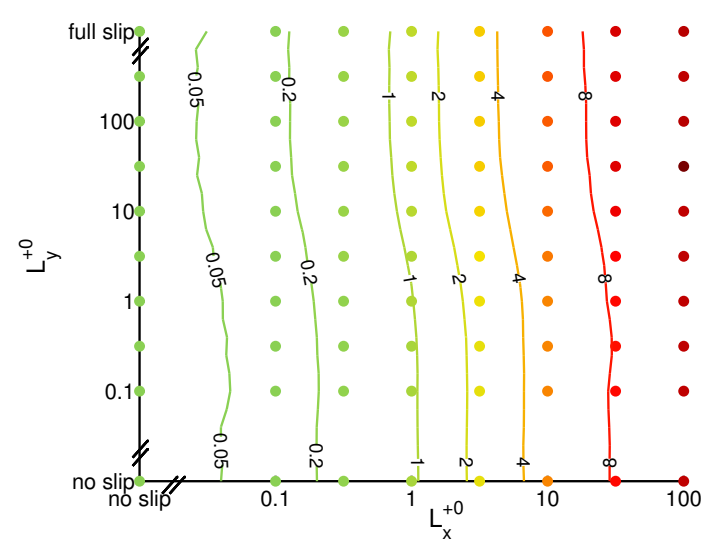

FIG. 5. The mean streamwise slip velocity on the wall $\left\langle u_{s}\right\rangle$ versus the streamwise and spanwise slip length, case $R e_{\tau_{0}}=180$.

(a)

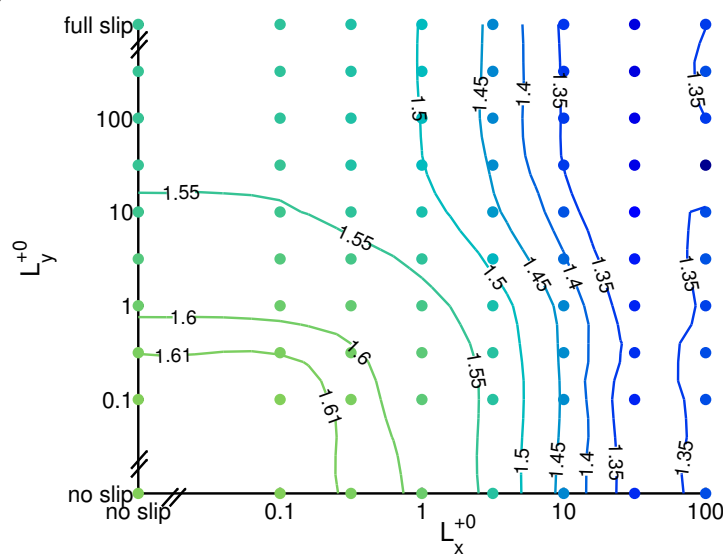

(b)

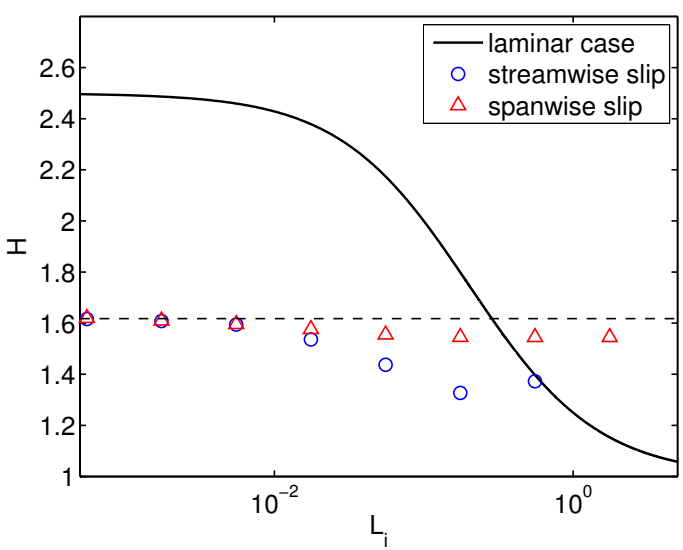

FIG. 6. Shape factor $H$ shown versus streamwise and spanwise slip length (a). The change in the shape factor for the purely streamwise (x-axis: $L_{i}=L_{x}$ ) and purely spanwise (x-axis: $L_{i}=L_{y}$ ) cases is compared to the shape factor dependence on the streamwise slip length in the laminar case in (b). The dashed line shows the value of the shape factor in the no-slip reference case. Case $R e_{\tau_{0}}=180$.

shown in figure 6, decreases with both increasing streamwise and spanwise slip length. In the case of increasing streamwise slip the decrease in the shape factor can be attributed to the upwards shift across the entire profile due to the finite slip velocity on the wall combined with the lower curvature of the profile across the channel that is needed to maintain a constant mass flow rate. The lower curvature of the profile is in line with the lower centreline velocity that is observed for increasing streamwise slip lengths (see figure 7). A (similar) decrease in the shape factor and the centreline velocity would also be expected in the corresponding laminar cases (see figure 6 (b)).

For very high streamwise slip lengths $\left(L_{x}^{+0}=100\right)$ the flow returns slowly to the laminar state. The shape factor approaches the value for the laminar solution $H \approx 1.4$ (see appendix $\mathrm{B}$ ) and is slightly larger than that of the next smaller streamwise slip length. This is due to the higher shape factor and the higher centreline velocity one would expect for the laminar case.

The decrease in the shape factor for increasing spanwise slip length is in comparison much smaller. Here, the decrease in the shape factor can be attributed to a higher slope in the near-wall region enforced by the higher wall-shear stress needed to balance the higher mean streamwise pressure gradient. This is accompanied by an increase of the centreline velocity. The Reynolds number $R e_{\tau}$ based on the actual wallshear stress increases with increasing spanwise slip length, and the observations made are in line with the expected Reynolds number dependence of the shape factor and the centreline velocity for turbulent channel flow ${ }^{16}$.

To give an overview of the change in the intensity of the rms velocity fluctuations the peak value of 
(a)

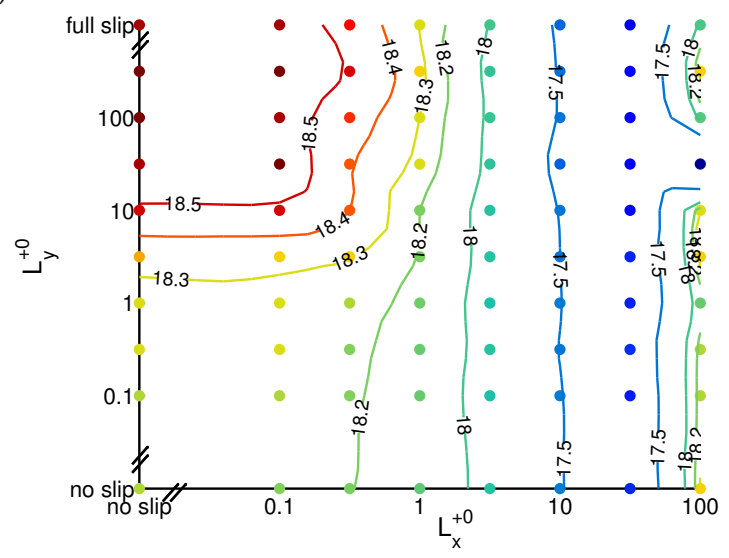

(b)

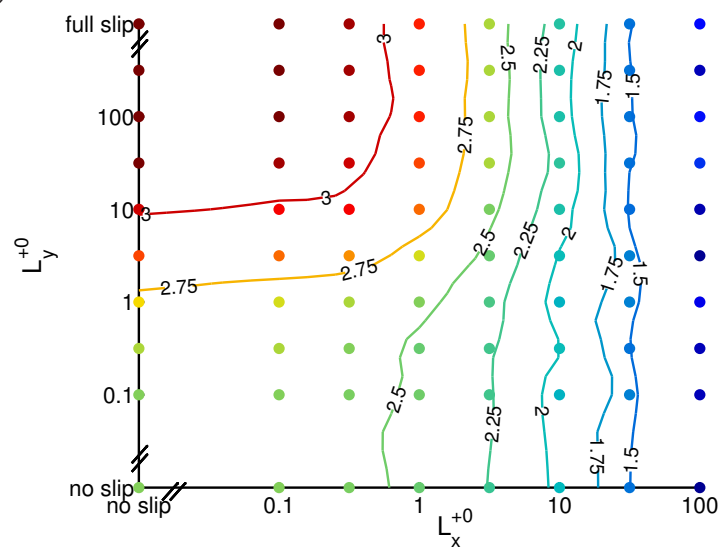

FIG. 7. Centreline velocity (a) and peak value of rms streamwise velocity fluctuations (b) shown versus streamwise and spanwise slip length, $R e_{\tau_{0}}=180$.

(a)

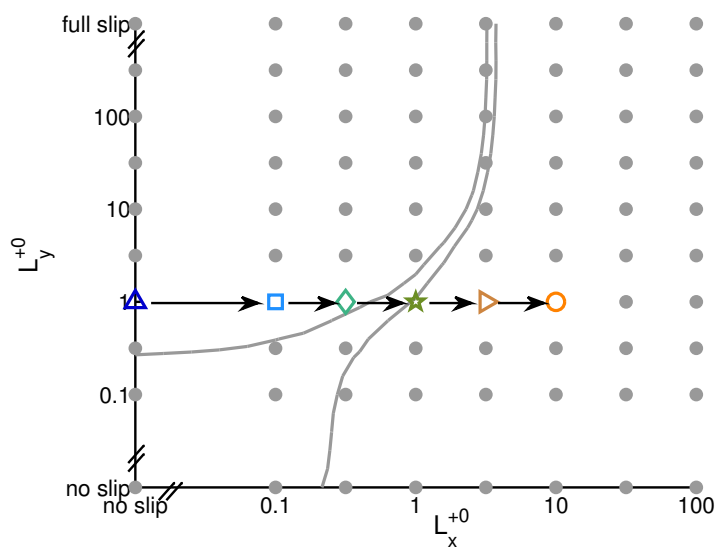

(b)

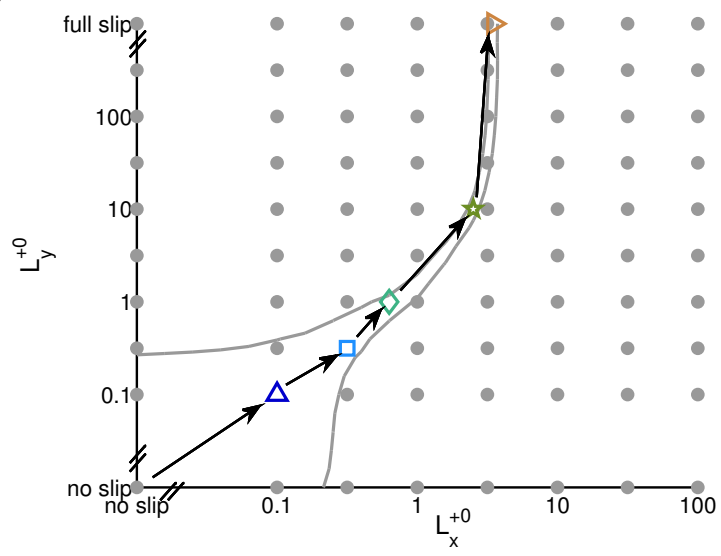

FIG. 8. Illustration of the two routes taken across the slip length map; (a): 'west-to-east'-direction, (b): along neutral curve. The colours and markers correspond to the colours and markers of the lines in the corresponding plots of the mean and rms velocity profiles (see figures 9 and 10).

the rms streamwise velocity fluctuations is shown in figure 7 (b). A high spanwise slip length leads to an intensification of the turbulent fluctuations and the peak value moves closer to the wall (not shown). For full slip in the spanwise direction $\left\langle u^{\prime 2}\right\rangle^{1 / 2}$ has increased by approximately $20 \%$ compared to the no-slip reference case $\left\langle u^{\prime 2}\right\rangle^{1 / 2} \approx 2.6$. Note that this increase is much higher than the increase in the turbulent fluctuations one would expect based on the corresponding increase in the wall-shear stress based Reynolds number $R e_{\tau}$ $\left(R e_{\tau}=230\right.$ for $\left.L_{x}^{+o}=0, L_{y}^{+o}=\infty\right)$ (see e.g. Ref. 16). For increasing streamwise slip length the peak value of $\left\langle u^{\prime 2}\right\rangle^{1 / 2}$ decreases and the position of the peak moves further away from the wall. Comparing figures 7 (b) and 1 (a) one can notice that there is a close correlation between change in drag and the peak value of $\left\langle u^{\prime 2}\right\rangle^{1 / 2}$. For drag-increasing slip-length combinations an increase in the peak value of $\left\langle u^{\prime 2}\right\rangle^{1 / 2}$ can be observed, whereas drag-reducing combinations are accompanied by a decrease in the peak value of $\left\langle u^{\prime 2}\right\rangle^{1 / 2}$.

The mean velocity profiles and rms velocity fluctuations for the purely streamwise, spanwise and isotropic cases follow the results in Ref. 6 well and will not be presented here. Instead the influence of the slip-length boundary condition will be illustrated by traversing the slip length map along two different routes shown in figure 8 that are of particular interest for the transition from the drag-increasing to the drag-reducing part of the slip length map. In the first case the slip length map is traversed in a 'west-to-east' direction by keeping a constant finite spanwise slip length $L_{\mathrm{y}}^{+0}=1$ and varying the streamwise slip length from $L_{x}^{+0}=0$ to $L_{x}^{+0}=10$, going from a case with a clear drag increase of $\approx 11 \%\left(L_{x}^{+0}=0, L_{y}^{+0}=1\right)$ to a case with a strong drag reduction of $\approx 49 \%\left(L_{x}^{+o}=10, L_{y}^{+o}=1\right)$. Figure 9 shows that for vanishing or very low streamwise slip length the mean streamwise velocity profile remains unchanged. With increasing streamwise slip length 
(a)

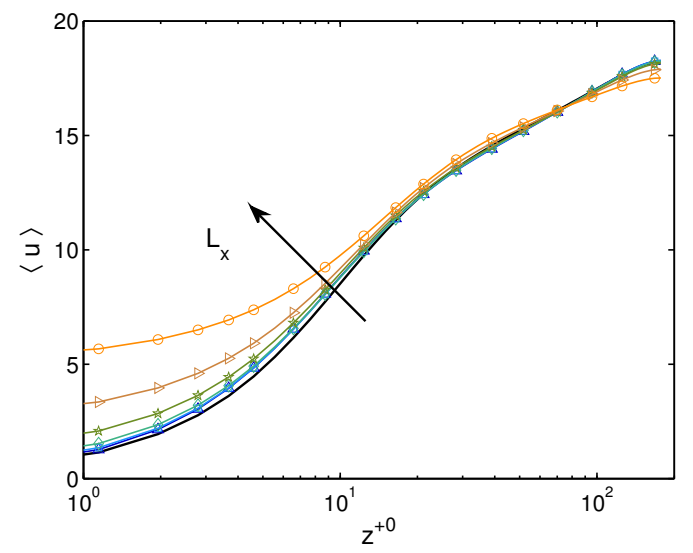

(b)

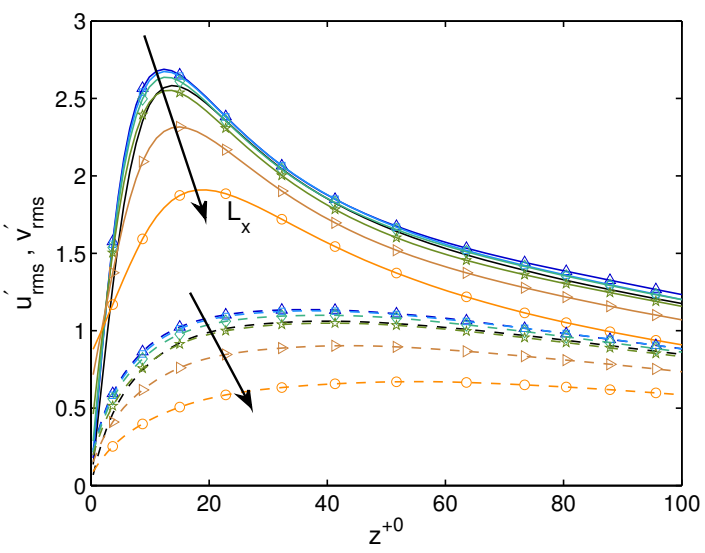

FIG. 9. The mean streamwise velocity profile (a) and the rms velocity fluctuations (b) of the streamwise (continuous lines) and spanwise (dashed lines) velocity components for the 'west-to-east'-traversal of the slip length map. Case $R e_{\tau_{0}}=180$. The colours and markers of the lines correspond to the colours and markers used in the illustration of the 'west-to-east' route across the slip length map in figure 8 (a). The black lines correspond to the no-slip reference case.

TABLE III. Slip-length combinations and corresponding percentage change in drag for the route along the neutral curve across the slip length map for $R e_{\tau_{0}}=180$.

\begin{tabular}{llllll}
\hline \hline$L_{x}^{+0}$ & 0.1 & 0.316 & 0.631 & 2.51 & 3.5 \\
$L_{y}^{+0}$ & 0.1 & 0.316 & 1 & 10 & $\infty$ \\
$\Delta \mathrm{D}$ & -0.1 & -1.2 & 0.8 & 0.7 & 0.1 \\
\hline \hline
\end{tabular}

the inner part of the mean streamwise velocity profile begins to shift upwards to accommodate the finite value of the slip velocity on the walls, whereas the outer part of the profile remains almost unchanged. The profile changes across the whole height of the channel only for the highest slip length considered. As the overall mass flow rate is kept constant, the small increase in mass flow rate near the walls due to the finite slip velocity on the walls does not affect the mean flow in the middle of channel. The velocity in the middle of the channel is decreased significantly only in cases where a very high slip velocity on the walls leads to a large increase in the mass flow near the walls. The rms velocity fluctuations show an increase across the full width of the channel for the drag-increasing cases at low streamwise slip lengths. With increasing streamwise slip length the intensity of the fluctuations decreases everywhere, except in the near-wall region, where an increase in the streamwise velocity fluctuations can be observed due to the increasing slip velocity on the wall (see also Ref. 6).

In the second case a route that approximately traces the neutral curve is followed across the slip length map. In order to achieve this three more simulations at intermediate slip-length combinations have been conducted. The slip length values and the corresponding percentage change in drag considered here are listed in table III. In all cases the change in drag is very low and does not exceed $2 \%$. Again the mean velocity profile shown in figure 10 is mainly influenced by the value of the streamwise slip length. As the streamwise slip length does not exceed $L_{x}^{+_{0}}=3.5$ only the inner region of the velocity profile is significantly affected whereas the outer region is approximately unchanged in all cases considered here. The level of rms velocity fluctuations is approximately the same in all cases. A significant change in the shape of the profile can be observed only near the walls where the effects of a finite slip velocity on the walls are felt. For the combinations involving large spanwise slip lengths the significant slip velocities on the walls lead to a shift of the peak of the $u_{\mathrm{rms}}$-fluctuations closer to the wall and a flattening of the profile of the $v_{\mathrm{rms}}$-fluctuations.

A possible interpretation of these results is that even for infinite spanwise slip the $v_{\text {rms }}$-fluctuations on the wall do not exceed the peak value of the corresponding profile. Therefore, once a rms spanwise slip velocity of approximately the peak value $\max \left(v_{\mathrm{rms}}\right)$ is reached a further increase of the spanwise slip length will have little effect. Thus the adverse effects of spanwise slip on the drag are limited. On the other hand, a streamwise slip length will always have a reducing effect on the intensity of the turbulent fluctuations, and this effect will increase with increasing slip lengths. Therefore a finite streamwise slip length is sufficient to 
(a)

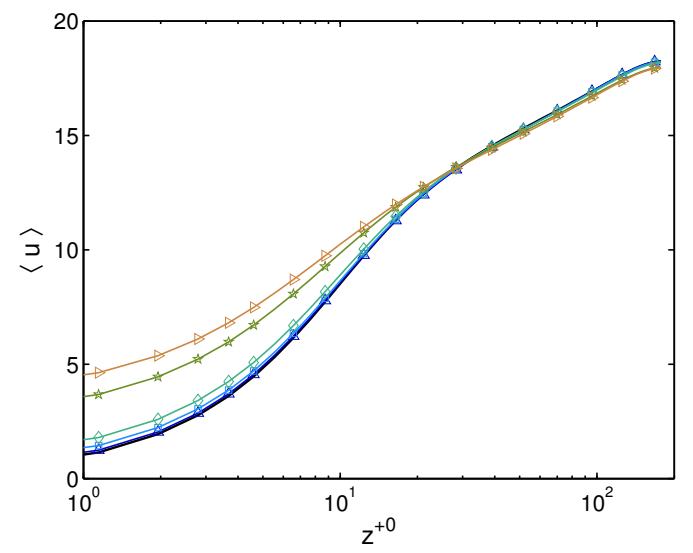

(b)

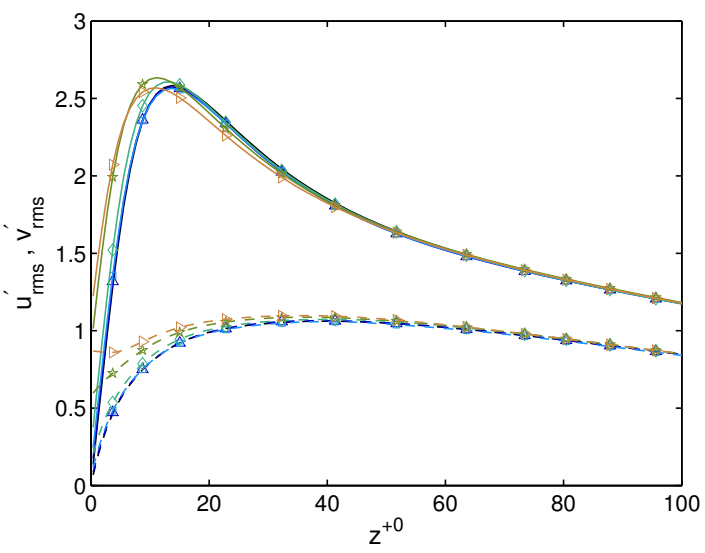

FIG. 10. The mean streamwise velocity profile (a) and the rms velocity fluctuations (b) of the streamwise (continuous lines) and spanwise (dashed lines) velocity components for the traversal of the slip length map along the neutral curve. Case $R e_{\tau_{0}}=180$. The colours and markers of the lines correspond to the colours and markers used in the illustration of the route along the neutral curve across the slip length map in figure 8 (b). The black lines correspond to the no-slip reference case.

overcome the turbulence-intensifying effects of infinite spanwise slip.

\section{WALL-SHEAR STRESS STATISTICS}

The statistics of the wall-shear stress are of special interest as the local wall-shear stress is directly involved in the specification of the Navier-slip boundary condition. The mean streamwise wall-shear stress balances the mean streamwise pressure gradient and thus its slip-length dependence can be inferred from the change in drag shown in section III, and will not be discussed here again. The application of a slip-length boundary condition changes also the intensity and distribution of the wall-shear stress fluctuations. Thus the fluctuating wall-shear stress is a useful diagnostic to study the effect of the slip-length boundary condition on near-wall turbulence. We will first discuss the changes in the streamwise wall-shear stress fluctuations before considering the effects of the slip-length boundary condition on the spanwise wall-shear stress.

\section{A. Streamwise wall-shear stress fluctuations}

Due to the fluctuations in the near-wall velocity, much higher shear stress values on the wall can be attained locally. In the standard no-slip case the ratio between the rms value of the streamwise wallshear stress fluctuations and the mean streamwise wall-shear stress $\left\langle\tau_{w, x}^{\prime 2}\right\rangle^{1 / 2} /\left\langle\tau_{w, x}\right\rangle$ is found to be between approximately 0.35 and 0.41 in experiments at moderate Reynolds numbers (see Refs 17-19) and is known to increase slowly with increasing Reynolds number (see e.g. Refs 16 and 20).

For the no-slip reference case we obtain a value of 0.36 which is in good agreement with the value of the DNS by Kim et al. ${ }^{21}(0.36)$. In figure 11 the ratio $\left\langle\tau_{w, x}^{\prime 2}\right\rangle^{1 / 2} /\left\langle\tau_{w, x}\right\rangle$ is plotted versus streamwise and spanwise slip length. The normalised streamwise wall-shear stress fluctuations decrease with increasing streamwise slip length. This is in line with the picture of an increasing laminarisation of the flow, since the wall-shear stress fluctuations would vanish for a laminar channel flow. The streamwise slip length hampers the build-up of extremely high wall-shear stress events, since the velocity on the wall can follow the near-wall velocity. This would not be possible in the standard no-slip case where the wall velocity is independent of the near-wall velocity, i.e. always vanishes.

The spanwise slip length has an opposite effect: the ratio $\left\langle\tau_{w, x}^{\prime 2}\right\rangle^{1 / 2} /\left\langle\tau_{w, x}\right\rangle$ increases with increasing spanwise slip length. The absolute increase in the streamwise wall-shear stress fluctuations is even higher, since the mean streamwise wall-shear stress increases with increasing spanwise slip length as well. The spanwise slip length therefore clearly leads to an intensification of the turbulence near the wall. As in the case of the peak value of the rms streamwise velocity fluctuations the streamwise wall-shear stress fluctuations correlate 


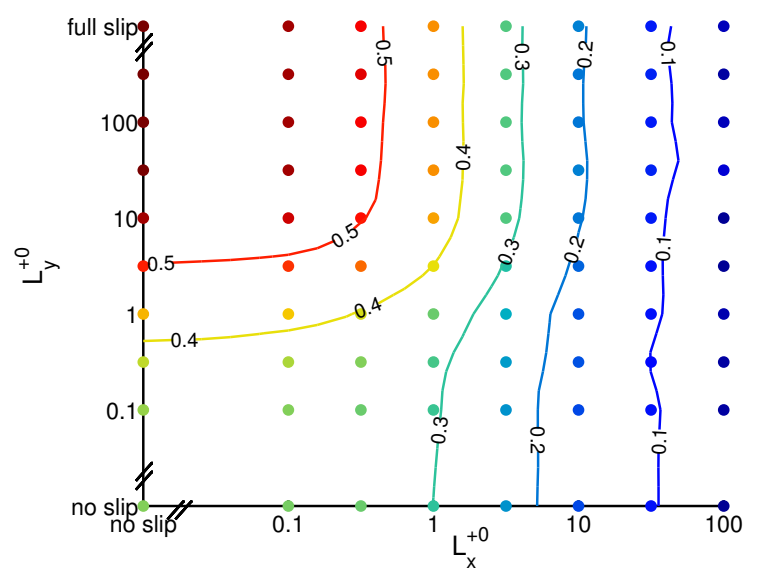

FIG. 11. Normalised streamwise wall-shear stress fluctuations $\left\langle\tau_{w, x}^{\prime 2}\right\rangle^{1 / 2} /\left\langle\tau_{w, x}\right\rangle$ versus streamwise and spanwise slip length, $R e_{\tau_{0}}=180$.

(a)

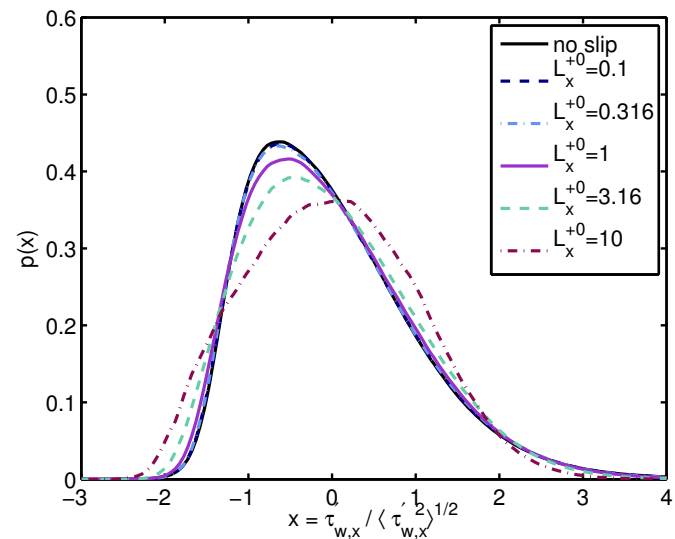

(b)

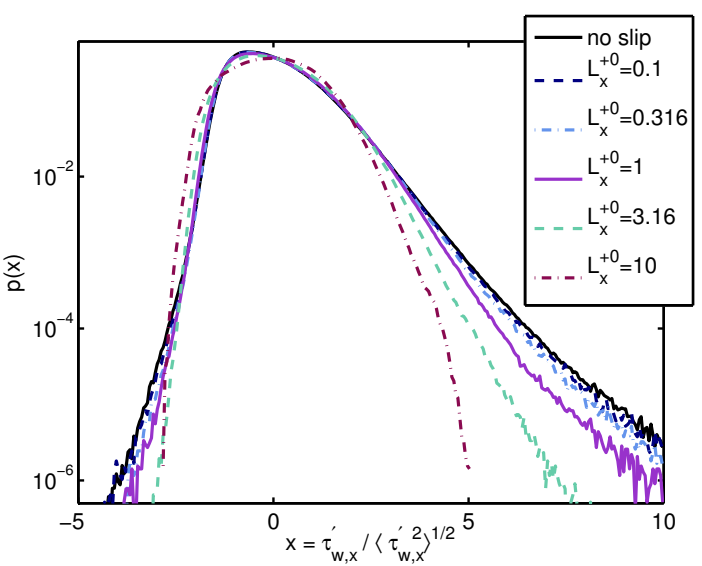

FIG. 12. Probability density function of streamwise wall-shear stress fluctuations for purely streamwise slip; (a): probability shown on a linear scale, (b): on a logarithmic scale; $R e_{\tau_{0}}=180$.

well with the change in drag. Along the neutral curve $\left\langle\tau_{w, x}^{\prime 2}\right\rangle^{1 / 2} /\left\langle\tau_{w, x}\right\rangle$ is approximately constant, an increase in drag is accompanied by an increase of $\left\langle\tau_{w, x}^{\prime 2}\right\rangle^{1 / 2} /\left\langle\tau_{w, x}\right\rangle$, and the opposite holds for a decrease in drag.

The probability density function (pdf) of the streamwise wall-shear stress fluctuations shown in figure 12 has a characteristically skewed shape and is also quite flat. In experiments and numerical simulations (under no-slip boundary conditions) skewness factors between 0.84 and 1.1, and flatness factors ranging from 4.1 to about 4.8 , have been measured ${ }^{18}$. The flatness of the wall-shear stress pdf is also known to increase slowly with the Reynolds number ${ }^{16,20}$.

The values obtained for the no-slip reference case in our simulation fall well within these ranges (skewness factor: $\mathcal{S} \approx 0.97$ and flatness factor $\mathcal{K} \approx 4.5)$. The streamwise slip-length boundary condition has a significant effect on the shape of the pdf of the streamwise wall-shear stress fluctuations. With increasing streamwise slip length the skewness of the pdf decreases and it becomes increasingly less flat (see figure 13). The decrease in skewness and flatness indicates that high (positive) wall-shear stress events are preferentially damped by the streamwise slip boundary condition as can also be observed from the decrease in the right hand tail of the pdf. For high streamwise slip length values the flatness factor drops below 3 and the skewness factor approaches zero, making the pdf sub-Gaussian.

The spanwise slip length has a smaller effect on the shape of the streamwise wall-shear stress pdf. Here an increase of the flatness and the skewness can be observed for moderate slip length values which approximately saturates for higher slip length values (see figure $13(\mathrm{~b})$ ).

We can infer from the wall-shear stress pdf in the no-slip reference case that negative values of the wall-shear stress can occur, since the pdf of the wall-shear stress fluctuations shows a finite signal for 
(a)

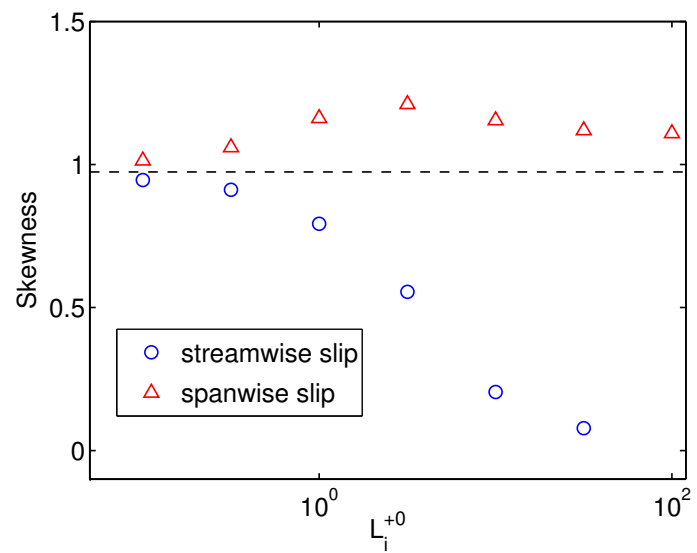

(b)

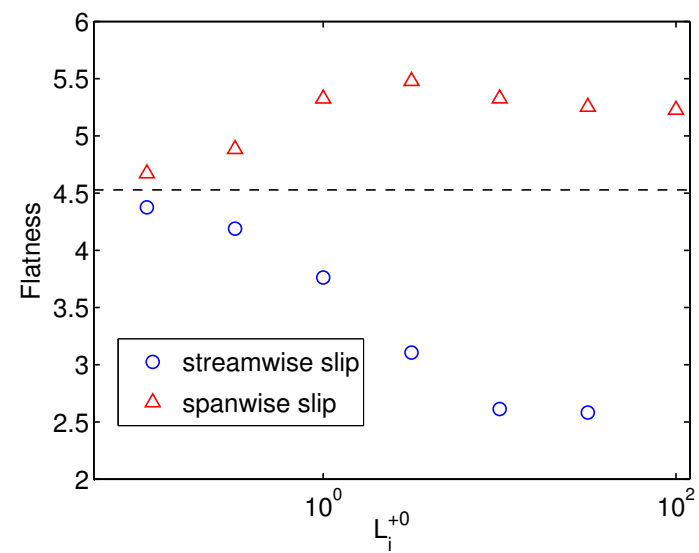

FIG. 13. Skewness (a) and flatness (b) factors of the streamwise wall-shear stress fluctuations for the purely streamwise (x-axis: $\left.L_{i}^{+0}=L_{x}^{+0}\right)$ and purely spanwise (x-axis: $L_{i}^{+0}=L_{y}^{+0}$ ) slip configurations versus slip length. $R e_{\tau_{0}}=180$.

(a)

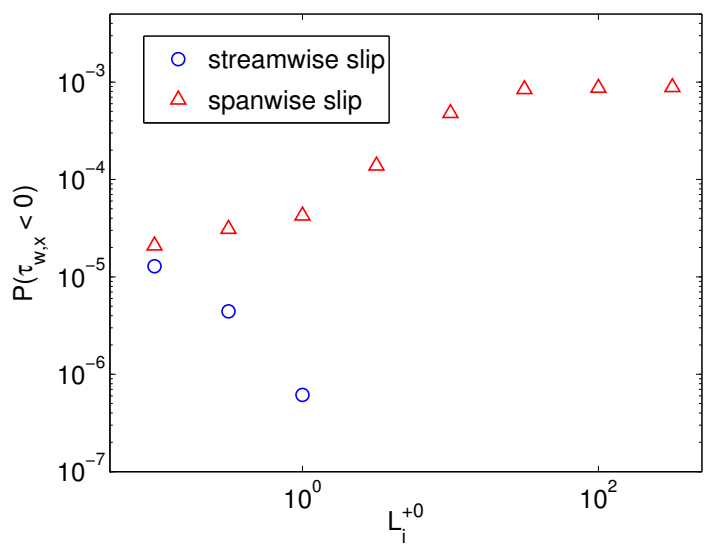

(b)

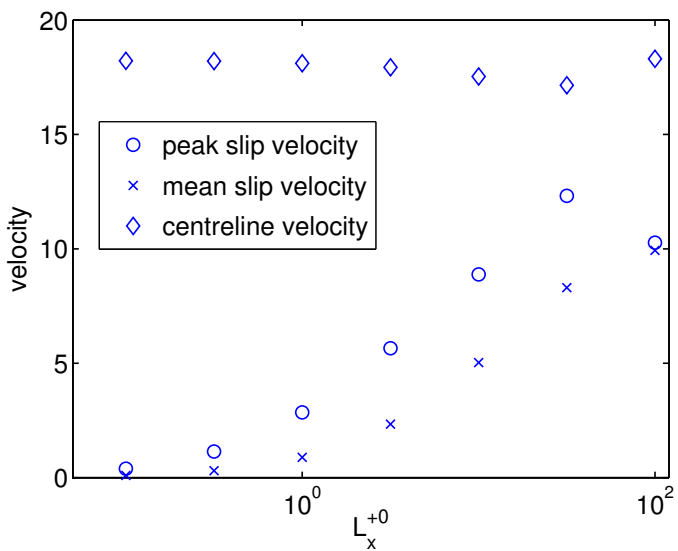

FIG. 14. (a): probability of negative streamwise wall-shear stress for the purely streamwise $\left(\mathrm{x}\right.$-axis: $\left.L_{i}^{+0}=L_{x}^{+0}\right)$ and purely spanwise (x-axis: $L_{i}^{+0}=L_{y}^{+0}$ ) slip-length combinations. (b): slip velocities $U_{s}^{\text {peak }},\left\langle u_{s}\right\rangle$ and centreline velocity versus slip length in the purely streamwise slip case. Case $R e_{\tau_{0}}=180$.

$x<-\frac{\left\langle\tau_{w, x}\right\rangle}{\left\langle\tau_{w, x}^{\prime 2}\right\rangle^{1 / 2}}=-2.8$. This corresponds to very rare events of negative streamwise velocity near the wall. By integrating the probability density function $p(x)$ of the streamwise wall-shear stress fluctuations from $-\infty$ (here due to the finite width of the resolution of the pdf: -10$)$ to $\xi=-\frac{\left\langle\tau_{w, x}\right\rangle}{\left\langle\tau_{w, x}^{\prime 2}\right\rangle^{1 / 2}}$ we can obtain a rough estimate for the probability of negative streamwise wall-shear stress events

$$
P\left(\tau_{w, x}<0\right)=\int_{-\infty}^{\xi} p(x) d x .
$$

For purely spanwise slip $P\left(\tau_{w, x}<0\right)$ corresponds to the probability of negative streamwise velocity near the wall. In the no-slip reference case we obtain a value of $P\left(\tau_{w, x}<0\right) \approx 2 \cdot 10^{-5}$ at $R e_{\tau_{0}}=180$. This is lower than values reported in the literature for the probability of negative wall-shear stress from DNS using spectral methods with Chebyshev polynomials in the wall-normal direction ${ }^{16,22}$. For an accurate computation of this quantity a very high near-wall resolution (as e.g. provided by Chebyshev methods) is needed, as the probability of reverse flow increases significantly below $z^{+}=1$ (see Ref. 22). Nevertheless it is interesting to consider the qualitative changes in this quantity for large slip lengths in the purely streamwise and spanwise slip cases.

The probability of negative wall-shear stress events decreases with increasing streamwise slip length (due to finite resolution of the pdf no events have been found for $L_{x}^{+t_{0}}>1$ ) and increases with increasing spanwise slip length (see figure $14(\mathrm{a})$ ). In the purely spanwise slip case the probability of negative streamwise velocities 
(a)

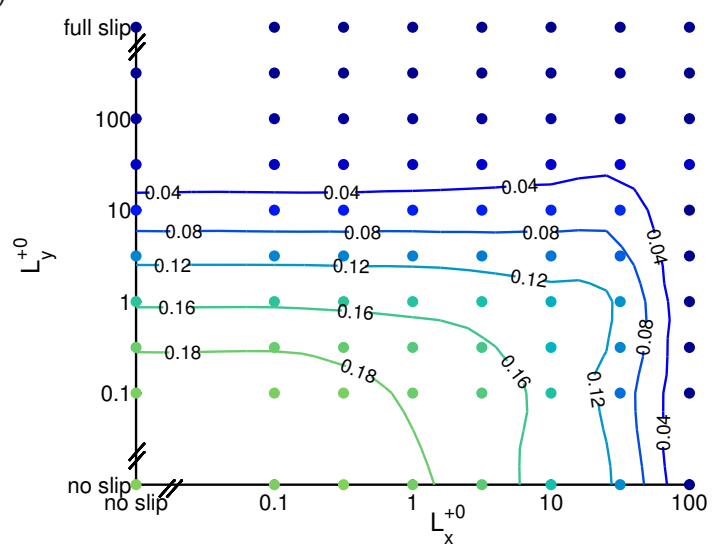

(b)

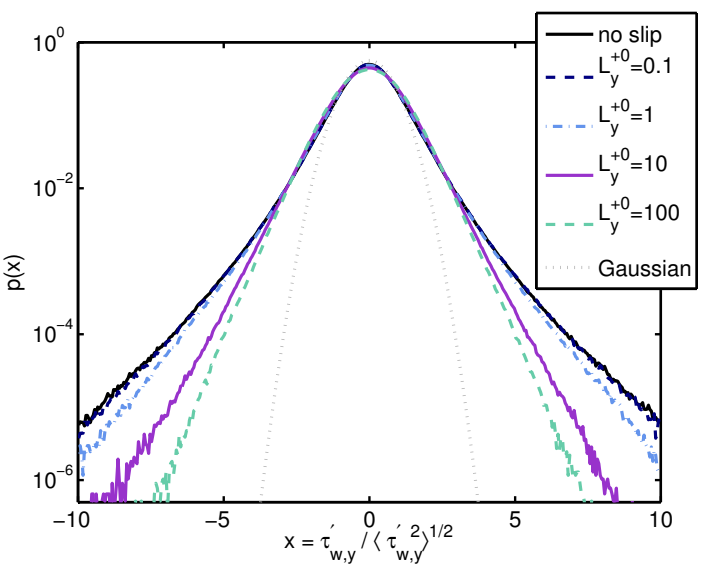

FIG. 15. (a): normalised spanwise wall-shear stress fluctuations $\left\langle\tau_{w, y}^{\prime 2}\right\rangle^{1 / 2} /\left\langle\tau_{w, x}\right\rangle$ versus streamwise and spanwise slip length. (b): probability density function of spanwise wall-shear stress fluctuations, the streamwise slip length is zero in all cases shown. $R e_{\tau_{0}}=180$.

near the wall is significantly enhanced. For infinite spanwise slip combined with vanishing streamwise slip we obtain $P\left(\tau_{w, x}<0\right) \approx 1 \cdot 10^{-3}$ which is an order of magnitude higher than the probability of negative wall-shear stress in the no-slip reference case. To reach a comparable probability of negative wall-shear stress in the no-slip case a Reynolds number in excess of $R e_{\tau}=1440$ would need to be attained ${ }^{16}$.

The strong increase in $P\left(\tau_{w, x}<0\right)$ for high spanwise slip is not caused by an increase in the left hand tail of the pdf of the wall-shear stress $p\left(\tau_{w, x}^{\prime} /\left\langle\tau_{w, x}^{\prime 2}\right\rangle^{1 / 2}\right)$. The significant growth in the probability of negative streamwise wall-shear stress with increasing spanwise slip can be attributed to the relative increase of the intensity of the streamwise wall-shear stress fluctuations $\left\langle\tau_{w, x}^{\prime 2}\right\rangle^{1 / 2}$ compared to the mean streamwise wall shear stress $\left\langle\tau_{w, x}\right\rangle$. The mean streamwise wall-shear stress is thus more easily overcome by the fluctuating streamwise wall-shear stress. In the computation of $P\left(\tau_{w, x}<0\right)$ this leads to a decrease of the ratio $\frac{\left\langle\tau_{w, x}\right\rangle}{\left\langle\tau_{w, x}^{\prime 2}\right\rangle^{1 / 2}}$ and thus an increase of the right boundary $\xi$ of the domain of integration in eqn (8).

At the other extreme of the wall-shear stress pdf, corresponding to very high positive values, the peak values of the streamwise slip velocity on the wall are of interest. As there is no clear maximum value, an estimate for the peak value is computed from

$$
U_{s}^{\text {peak }}=L_{x}^{+0}\left(x^{*}\left\langle\tau_{w, x}^{\prime 2}\right\rangle^{1 / 2}+\left\langle\tau_{w, x}\right\rangle\right)
$$

where the peak streamwise wall-shear stress fluctuation

$$
x^{*}=\left\{\min (x) \mid p(x)<10^{-5} \cap x>0\right\}
$$

is based on an arbitrary cut-off value $10^{-5}$ for the pdf. In the case of small streamwise slip length the peak value can be considerably higher than the mean value of the slip velocity on the wall (see figure 14 (b)). With increasing slip length the relative difference between the peak and the mean value decreases. For higher streamwise slip length the peak value starts to approach the centreline velocity. However, at the highest slip length investigated (this is a case that is in the process of laminarisation) this trend is reversed as the $U_{s}^{\text {peak }}$ becomes equal to the mean slip velocity on the wall.

\section{B. Spanwise wall-shear stress fluctuations}

Spanwise wall-shear stress fluctuations have received far less attention in the literature than the streamwise wall-shear stress. The level of the spanwise rms wall-shear stress fluctuations is approximately $1 / 2$ of the value of the streamwise rms wall-shear stress fluctuations ${ }^{17}$ in the standard no-slip case. The normalised rms spanwise wall-shear stress fluctuation $\left\langle\tau_{w, y}^{\prime 2}\right\rangle^{1 / 2} /\left\langle\tau_{w, x}\right\rangle$ is found to be 0.19 in the no-slip reference case, which is in good agreement with the value of Kim et al. ${ }^{17,21}$ (also 0.19). The level of the normalised spanwise wall-shear stress fluctuations, shown in figure 15, decreases both with increasing spanwise and increasing streamwise slip length. At first sight this may be surprising, since the streamwise and spanwise slip lengths 
(a)

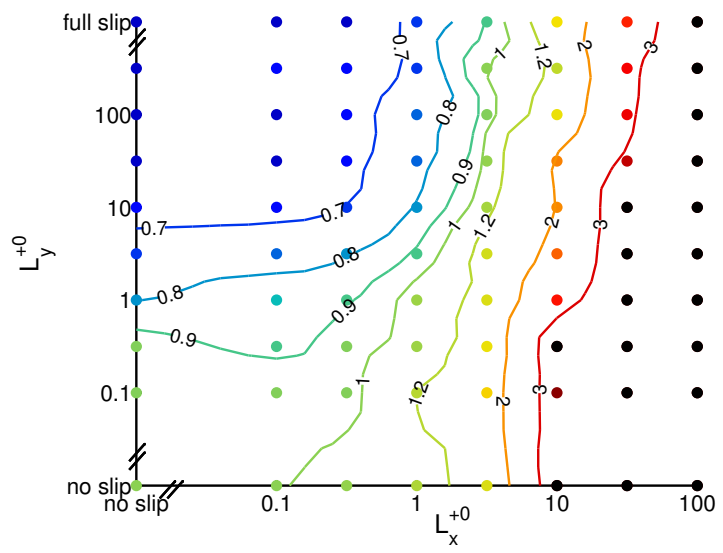

(b)

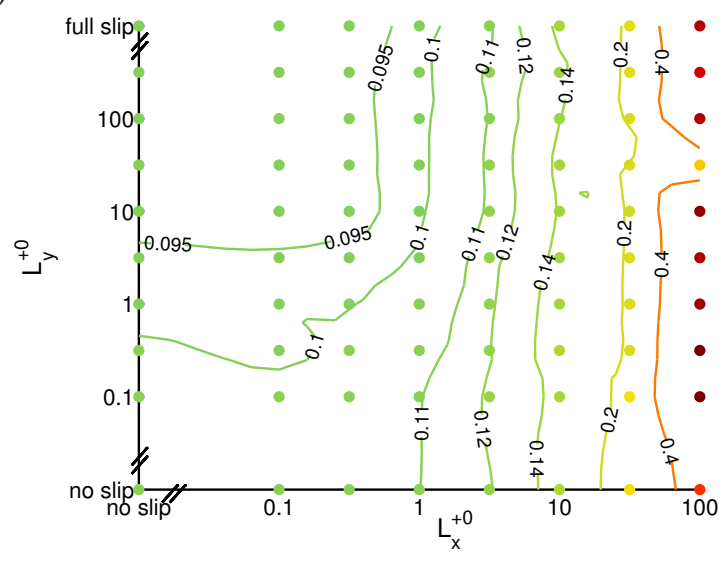

FIG. 16. Streamwise (a) and spanwise (b) correlation length scales of the streamwise velocity at a distance of $z^{+0} \approx 10$ from the wall shown versus streamwise and spanwise slip lengths. Case $R e_{\tau_{0}}=180$.

show opposite effects for the streamwise wall-shear stress fluctuations, but the decrease of the spanwise wall-shear stress fluctuations can be attributed to two different processes. Firstly, for increasing streamwise slip length the overall level of turbulent fluctuations decreases due to an increasing regularisation of the flow and therefore the level of spanwise wall-shear stress fluctuations decreases as well. Secondly, for increasing spanwise slip length the spanwise slip boundary condition prevents the build-up of very high wall-shear stress values, since the spanwise velocity on the wall can follow the near-wall fluctuations of the spanwise velocity. Thus the overall level of the fluctuations decreases. The fact that extreme events are preferentially damped in the case of high spanwise slip length values can also be observed from the pdf of the spanwise wall-shear stress fluctuations (see figure $15(\mathrm{~b})$ ). In the no-slip reference case this is quite flat $(\mathcal{K} \approx 6.3)$, however with increasing spanwise slip length the flatness decreases $\left(\mathcal{K} \approx 3.7\right.$ for $\left.L_{x}^{+o}=0, L_{y}^{+0}=316\right)$.

For vanishing or low streamwise slip high spanwise slip has a strong effect on small-scale turbulence in the near-wall region by significantly enhancing the anisotropy of the turbulence. In this case strong and highly intermittent streamwise wall-shear stress fluctuations are combined with weak and close to Gaussian spanwise wall-shear stress fluctuations. The spanwise slip-length boundary condition is therefore able to change the character of the small-scale turbulence in the near-wall region.

\section{FLOW STRUCTURE}

In this section we consider to which extent the Navier-slip boundary condition changes the structure of the flow, focusing on the characteristic streaks in the near-wall region. To give an overview of the effects of various streamwise spanwise slip-length combinations, streamwise and spanwise correlation length scales of the streamwise velocity are shown in figure 16 for a distance of $z^{+_{0}} \approx 10$ from the wall. The streamwise and spanwise slip lengths have been computed based on the point where the corresponding correlation functions $R_{u, x x}$ or $R_{u, y y}$ fall the first time below $1 / e$, i.e. for the streamwise correlation length scale

$$
L_{u, x x}=\left\{\min (\Delta x) \mid R_{u, x x}(\Delta x)<\frac{1}{e}\right\}
$$

where

$$
R_{u, x x}(\Delta x)=\frac{\left\langle u^{\prime}(x, y, z) u^{\prime}(x+\Delta x, y, z)\right\rangle}{\left\langle u^{\prime 2}(x, y, z)\right\rangle} .
$$

This measure has been chosen as it is less sensitive to the computational box size than the integral length scale. For high streamwise slip the streamwise correlation length scale is not always defined, since the structures in the velocity field grow beyond the domain size of the simulation. The corresponding cases are indicated by black dots on the map. This is consistent with an eventual return to the laminar case for very high streamwise slip lengths as the decay of streamwise streaks is one of the last steps of relaminarisation ${ }^{14,23}$. The streamwise correlation length $L_{u, x x}$ can be seen as a measure for the length of the streaks and the 
(a)

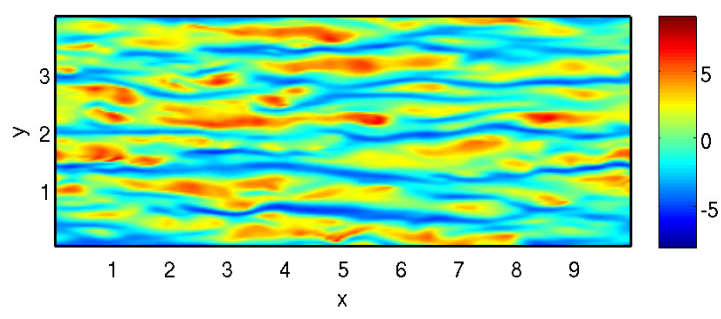

(c)

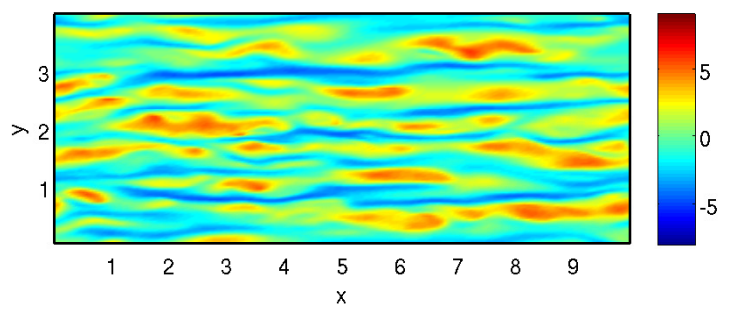

(b)

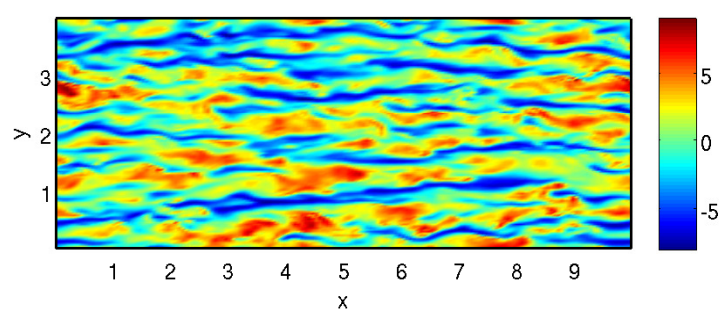

(d)

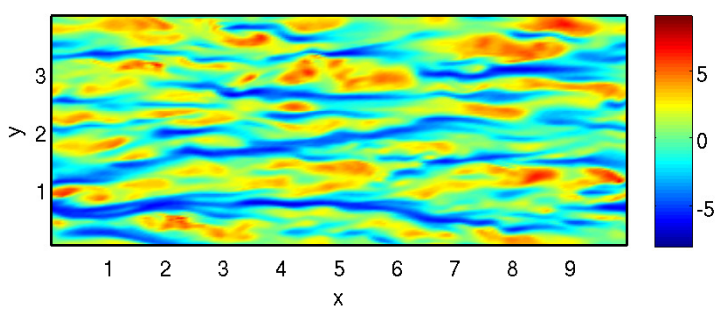

FIG. 17. Contours of streamwise velocity fluctuations at a distance of $z^{+_{0}} \approx 10$ from the wall. (a): no-slip case, (b): $L_{x}^{+0}=0, L_{y}^{+0}=316,(\mathrm{c}): L_{x}^{+0}=3.16, L_{y}^{+0}=0,(\mathrm{~d}): L_{x}^{+0}=3.16, L_{y}^{+0}=316$. Case $R e_{\tau_{0}}=180$.

spanwise correlation length for the width of the streaks in the near wall region. Increasing spanwise slip length leads to a decrease in $L_{u, x x}$ and to a small decrease in $L_{u, y y}$. In a slice through the streamwise velocity field at a distance of $z^{+0} \approx 10$ from the wall we can observe that the streaks tend to be disrupted and appear more irregular than in the no-slip reference case (see figure 17). Increasing streamwise slip leads to an increase in $L_{u, x x}$ and $L_{u, y y}$ and the streaks become more regular than in the no-slip reference case. For moderate slip lengths the changes in the flow structure near the wall are not large. The streaky pattern can still be observed in all cases and the changes in the correlation length scales are comparatively small. The flow structure begins to change significantly only for very high streamwise slip lengths.

The streak amplitude increases with increasing spanwise slip and decreases with increasing streamwise slip. This is in agreement with the results for the peak streamwise velocity fluctuations discussed in section V.

In figure $18 L_{u, x x}$ and $L_{u, y y}$ are shown for the purely streamwise and spanwise slip cases for several distances from the wall. Near the wall a significant increase in $L_{u, x x}$ for increasing streamwise slip can be observed, whereas a decrease occurs for increasing spanwise slip, as has already been observed. For high distances from the wall the streamwise correlation lengths are close to the no-slip reference case. This indicates that the structure of the streamwise velocity in the outer layer is not strongly affected by presence of spanwise or moderate streamwise slip. The collapse onto the no-slip reference case is delayed to higher distances from the wall for higher slip lengths. In contrast, the effect of the slip-length boundary condition on the spanwise correlation length $L_{u, y y}$ is very small for both the purely streamwise and purely spanwise slip cases.

\section{CONCLUSIONS}

A systematic study of the effect of an anisotropic Navier-slip boundary condition on turbulent channel flow has been presented. Depending on the relative magnitude of the streamwise and spanwise slip lengths, both drag-reducing and drag-increasing cases can be observed. A threshold value of approximately three to four times the viscous length scale has been identified for the streamwise slip length, beyond which drag is reduced in all cases even for infinite spanwise slip. For purely spanwise slip the change in drag saturates for high slip lengths $L_{y}^{+0}=\mathcal{O}\left(10^{2}\right)$. Even infinite spanwise slip does not lead to a further increase in drag. At the moderate Reynolds numbers studied here the Reynolds number has only a small effect on the observed change in drag when the slip lengths are the same in units of the corresponding viscous length scale. The only Reynolds number effect observed is the slightly smaller increase in drag for high spanwise slip lengths at the higher Reynolds number.

A detailed comparison has been made with the implicit formula of Fukagata et al. ${ }^{89}$ for the relation of the change in drag with the streamwise and spanwise slip length. A new version for the fitting function contained in this model has been presented, which reduces the number of free parameters in the model from 
(a)

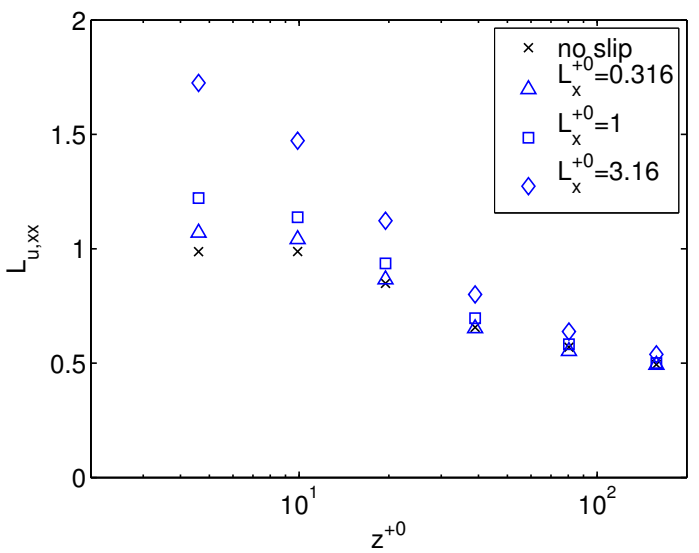

(c)

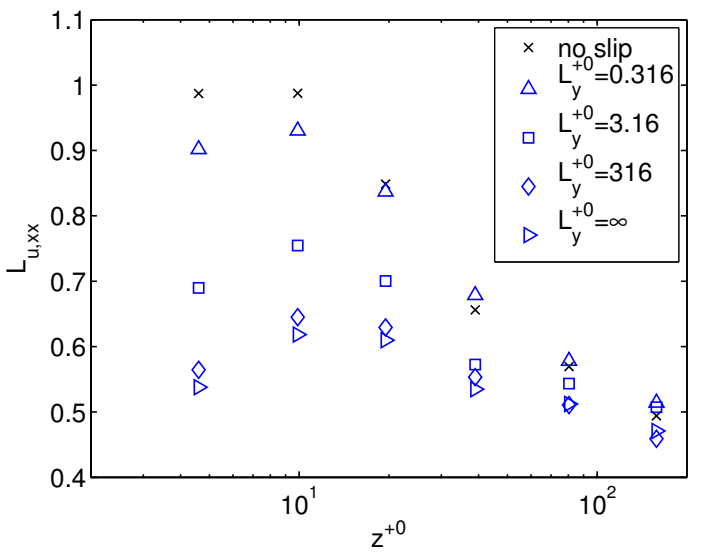

(b)

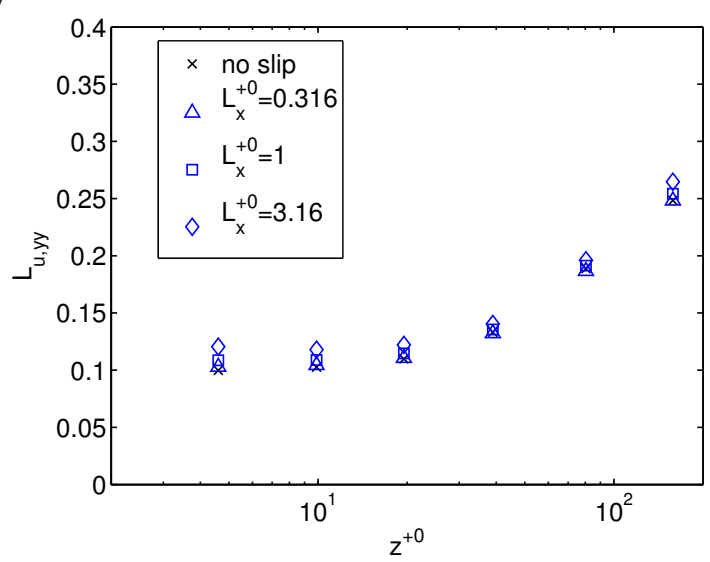

(d)

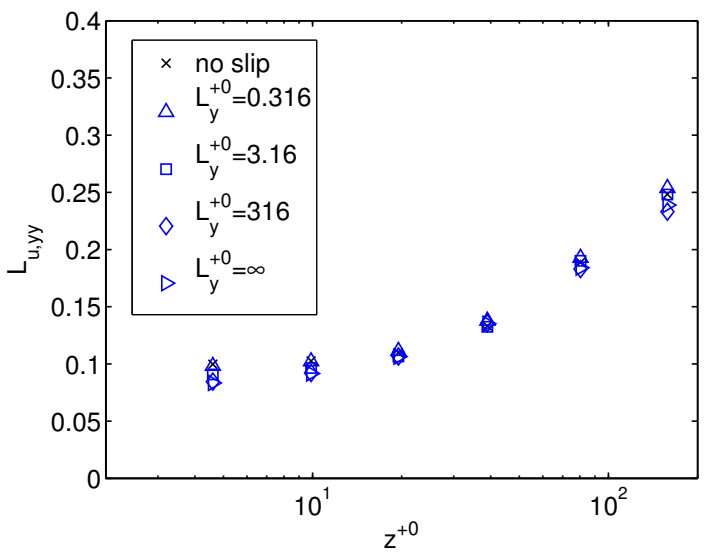

FIG. 18. Streamwise (a), (c) and spanwise (b), (d) correlation length scale of the streamwise velocity component for different distances from the wall. (a), (b) purely streamwise slip case; (c), (d): purely spanwise slip case. Case $\operatorname{Re}_{\tau_{0}}=180$.

four to two, has a simpler form than the original version of the fitting function and gives a more accurate representation for the neutral curve. For both versions a good agreement was found with the DNS data, implying that any coupling between the effects of the streamwise and spanwise slip does not have a strong influence on the change in drag.

The change in drag is shown to be correlated with both the amplitude of the near-wall peak of the streamwise velocity fluctuations and the streamwise wall-shear stress fluctuations. For slip-length combinations which lead to a negligible change in drag the level of the velocity fluctuations is unchanged compared to the no-slip reference case, except in the immediate vicinity of the wall.

A detailed investigation of the wall-shear stress statistics revealed an increase of the intensity of the streamwise wall-shear stress fluctuations with increasing spanwise slip length, whereas streamwise slip led to a decrease. This corresponds to a significant increase of the probability of backflow near the wall for the purely spanwise slip case for high spanwise slip lengths. The intensity and intermittency of the spanwise wall-shear stress fluctuations decrease with both increasing spanwise and increasing streamwise slip. High spanwise slip can therefore change the nature of near-wall turbulence, by enhancing the intensity and intermittency of the streamwise wall-shear stress fluctuations and decreasing the intensity and intermittency of the spanwise wall-shear stress fluctuations.

The changes introduced by the Navier-slip boundary condition into the structure of the velocity field have been investigated, focusing on the streamwise near-wall streaks in the velocity field. High spanwise slip leads to disrupted and more irregular near-wall streaks whereas high streamwise slip leads to straighter and more regular streaks. The effect of the Navier-slip boundary condition on the spanwise correlation length of the streamwise velocity is minimal for moderate streamwise slip lengths.

The description of superhydrophobic surfaces by a linear slip-length boundary condition with a constant slip length, as used in the present study, is only applicable for moderate shear rates ${ }^{24}$. In general, the slip 
TABLE IV. Influence of domain size and Reynolds number on the mean streamwise pressure gradient and the percentage change in drag for two representative slip length cases.

\begin{tabular}{ll|cccccc}
\hline \hline$L_{x}^{+0}$ & $L_{y}^{+0}$ & domain & $R e_{\tau_{0}}$ & $\left\langle\frac{d \bar{p}}{d x}\right\rangle$ & $\sigma\left(\left\langle\frac{d \bar{p}}{d x}\right\rangle\right)$ & $\Delta D$ & $\sigma(\Delta D)$ \\
\hline 0 & 0 & $5 \times 2 \times 2$ & 360 & -1.000 & 0.019 & & \\
0 & 0 & $10 \times 4 \times 2$ & 360 & -0.996 & 0.015 & & \\
0 & 0 & $10 \times 4 \times 2$ & 180 & -1.002 & 0.017 & & \\
0 & 1 & $5 \times 2 \times 2$ & 360 & -1.085 & 0.021 & 8.5 & 2.9 \\
0 & 1 & $10 \times 4 \times 2$ & 360 & -1.087 & 0.018 & 9.1 & 2.5 \\
0 & 1 & $10 \times 4 \times 2$ & 180 & -1.110 & 0.022 & 10.7 & 2.8 \\
1 & 0 & $5 \times 2 \times 2$ & 360 & -0.902 & 0.017 & -9.8 & 2.4 \\
1 & 0 & $10 \times 4 \times 2$ & 360 & -0.897 & 0.017 & -10.0 & 2.2 \\
1 & 0 & $10 \times 4 \times 2$ & 180 & -0.886 & 0.019 & -11.6 & 2.4 \\
\hline \hline
\end{tabular}

length will depend on the shear rate at the wall ${ }^{25}$. As the local instantaneous shear rate in a turbulent flow at the wall can significantly exceed the mean wall-shear rate, a shear-rate dependent non-linear slip-length boundary condition may lead to different changes in drag. Since no non-linear slip length model has yet been derived for superhydrophobic surfaces this will need to be a subject for future investigations.

\section{ACKNOWLEDGMENTS}

The support of the Engineering and Physical Sciences Research Council under grant number EP/G058318/1 and EP/G069581/1 is gratefully acknowledged.

\section{Appendix A: Influence of the domain size on the change in drag}

In order to ensure that the smaller domain size used for the $R e_{\tau_{0}}=360$ simulations does not influence the mean streamwise pressure gradient and the estimated value of the change in drag, two representative slip length cases and the reference case were run for both the full and the reduced domain size. The resulting values for the mean streamwise pressure gradient and the change in drag are given in table IV. The values of the mean streamwise pressure gradient for the full and the reduced domain size agree well with each other, with the differences being less than $1 \%$. The differences in the change in drag are comparatively larger but still considerably lower than the standard deviation $\sigma(\Delta D)$. We can conclude that the smaller domain size is sufficient to establish mean flow quantities such as the mean streamwise pressure gradient and the change in drag.

\section{Appendix B: Dependence of the shape factor $H$ on the slip length in the laminar case}

The shape factor $H=\delta^{*} / \theta$ is the ratio of the displacement thickness $\delta^{*}=\int_{0}^{1}\left(1-\frac{\langle u(z)\rangle}{\left\langle u_{c}\right\rangle}\right) d z$ to the momentum thickness $\theta=\int_{0}^{1} \frac{\langle u(z)\rangle}{\left\langle u_{c}\right\rangle}\left(1-\frac{\langle u(z)\rangle}{\left\langle u_{c}\right\rangle}\right) d z$, where $u_{\mathrm{c}}$ is the centreline velocity. It is used to characterise the 'flatness' of the mean velocity profile ${ }^{7}$, i.e. a lower shape factor corresponds to a flatter profile. For laminar channel flow the shape factor is $H=2.5$ under standard no-slip boundary conditions on the walls. When streamwise slip boundary conditions are applied on the walls the shape factor varies with the slip length

$$
H\left(L_{x}\right)=\frac{5}{2}\left(\frac{1+2 L_{x}}{1+5 L_{x}}\right) .
$$

The spanwise slip length $L_{y}$ has no effect on laminar channel flow as the spanwise velocity component vanishes. Relation (B1) shows that with increasing streamwise slip length $L_{x}$ the shape factor decreases; this 
means that the mean streamwise velocity profile gets increasingly flatter. In the limit of infinite streamwise slip, $L_{x} \rightarrow \infty$, the shape factor approaches its theoretical lower bound ${ }^{26} H=1$ for a perfectly flat profile.

${ }^{1}$ G. McHale, M. I. Newton, and N. J. Shirtcliffe, "Immersed superhydrophobic surfaces: Gas exchange, slip and drag reduction properties," Soft Matter 6, 714-719 (2010).

2 J. P. Rothstein, "Slip on superhydrophobic surfaces," Annual Review of Fluid Mechanics 42, 89-109 (2010).

${ }^{3}$ R. J. Daniello, N. E. Waterhouse, and J. P. Rothstein, "Drag reduction in turbulent flows over superhydrophobic surfaces," Physics of Fluids 21, 085103 (2009).

${ }^{4}$ R. S. Voronov, D. V. Papavassiliou, and L. L. Lee, "Review of fluid slip over superhydrophobic surfaces and its dependence on the contact angle," Industrial \& Engineering Chemistry Research 47, 2455-2477 (2008).

${ }^{5}$ B. Woolford, J. Prince, D. Maynes, and B. W. Webb, "Particle image velocimetry characterization of turbulent channel flow with rib patterned superhydrophobic walls," Physics of Fluids 21, 085106 (2009).

${ }^{6}$ T. Min and J. Kim, "Effects of hydrophobic surface on skin-friction drag," Physics of Fluids 16, L55-58 (2004).

${ }^{7}$ S. B. Pope, Turbulent Flows (Cambridge University Press, 2000).

${ }^{8}$ K. Fukagata, N. Kasagi, and P. Koumoutsakos, "A theoretical prediction of friction drag reduction in turbulent flow by superhydrophobic surfaces," Physics of Fluids 18, 051703 (2006)

${ }^{9}$ K. Fukagata, N. Kasagi, and P. Koumoutsakos, "Erratum: "a theoretical prediction of friction drag reduction in turbulent flow by superhydrophobic surfaces" [phys. fluids [bold 18], 051703 (2006)]," Physics of Fluids 18, 089901 (2006).

${ }^{10} \mathrm{~J}$. You, H. Choi, and J. Y. Yoo, "A modified fractional step method of keeping a constant mass flow rate in fully developed channel and pipe flows," KSME Int J 14, 547 (2000).

${ }^{11}$ D. A. Lockerby, J. M. Reese, D. R. Emerson, and R. W. Barber, "Velocity boundary condition at solid walls in rarefied gas calculations," Physical Review E 70, 017303 (2004).

${ }^{12}$ T. Min and J. Kim, "Effects of hydrophobic surface on stability and transition," Physics of Fluids 17, 108106 (2005).

${ }^{13}$ B. Eckhardt, T. M. Schneider, B. Hof, and J. Westerweel, "Turbulence transition in pipe flow," Annual Review of Fluid Mechanics 39, 447-68 (2007).

${ }^{14} \mathrm{O}$. Iida and Y. Nagano, "The relaminarization mechanisms of turbulent channel flow at low Reynolds numbers," Flow, Turbulence and Combustion 60, 193-213 (1998).

${ }^{15}$ R. B. Dean, "Reynolds number dependence of skin friction and other bulk flow variables in two-dimensional rectangular duct flow," Trans. ASME I: J. Fluids Engng. 100, 215 (1978).

${ }^{16}$ Z. W. Hu, C. L. Morfey, and N. D. Sandham, "Wall pressure and shear stress spectra from direct simulations of channel flow," AIAA J. 44, 1541-1549 (2006).

${ }^{17}$ P. H. Alfredsson, A. V. Johansson, J. H. Haritonidis, and H. Eckelmann, "The fluctuating wall-shear stress in the viscous sublayer," Physics of Fluids 31, 1026 (1988).

${ }^{18} \mathrm{~S}$. Obi, K. Inoue, T. Furukawa, and S. Masuda, "Experimental study on the statistics of wall shear stress in turbulent channel flows," International Journal of Heat and Fluid Flow 17, 187 (1996).

${ }^{19}$ N. Miyagi, M. Kimura, H. Shoji, A. Saima, C.-M. Ho, S. Tung, and Y.-C. Tai, "Statistical analysis on wall shear stress of turbulent boundary layer in a channel flow using micro-shear stress imager," International Journal of Heat and Fluid Flow 21, 576-581 (2000).

${ }^{20}$ R. Örlü and P. Schlatter, "On the fluctuating wall-shear stress in zero pressure-gradient turbulent boundary layer flows," Physics of Fluids 23, 021704 (2011).

${ }^{21}$ J. Kim, P. Moin, and R. Moser, "Turbulence statistics in fully developed channel flow at low Reynolds number," Journal of Fluid Mechanics 177, 133-166 (1987).

${ }^{22}$ P. Lenaers, Q. Li, G. Brethouwer, P. Schlatter, and R. Örlü, "Negative streamwise velocities and other rare events near the wall in turbulent flows," Journal of Physics: Conference Series 318, 022013 (2011).

${ }^{23}$ B. Cantwell, D. Coles, and P. Dimotakis, "Structure and entrainment in the plane of symmetry of a turbulent spot," Journal of Fluid Mechanics 87, 641-672 (1978).

${ }^{24}$ C.-H. Choi, K. J. A. Westin, and K. S. Breuer, "Apparent slip flows in hydrophilic and hydrophobic microchannels," Physics of Fluids 15, 2897-2902 (2003).

${ }^{25}$ J. Hyväluoma and J. Harting, "Slip over structured surfaces with entrapped microbubbles," Physical Review Letters 100, 246001 (2008).

${ }^{26}$ F. M. White, Viscous Fluid Flow (McGraw-Hill, 1991). 